\title{
Effects of kiwifruit extracts on colonic gene and protein expression levels in IL-10 gene-deficient mice
}

\author{
Shelley J. Edmunds ${ }^{1,2}$, Nicole C. Roy ${ }^{3,4}$, Marcus Davy ${ }^{5}$, Janine M. Cooney ${ }^{1}$, Matthew P. G. Barnett ${ }^{3}$, \\ Shuotun Zhu ${ }^{6}$, Zaneta Park ${ }^{7}$, Donald R. Love ${ }^{2}$ and William A. Laing ${ }^{1 *}$ \\ ${ }^{1}$ Food Innovation, Plant and Food Research Limited, Private Bag 92169, Auckland 1142, New Zealand \\ ${ }^{2}$ School of Biological Sciences, University of Auckland, Auckland, New Zealand \\ ${ }^{3}$ Food and Textiles Group, AgResearch Grasslands, Palmerston North, New Zealand \\ ${ }^{4}$ The Riddet Institute, Massey University, Palmerston North, New Zealand \\ ${ }^{5}$ Sustainable Production Group, Plant and Food Research Limited, Hamilton, New Zealand \\ ${ }^{6}$ Department of Nutrition, University of Auckland, Auckland, New Zealand \\ ${ }^{7}$ Bioinformatics, Mathematics and Statistics Section, AgResearch Grasslands, Palmerston North, New Zealand \\ (Submitted 24 May 2011 - Final revision received 15 August 2011 - Accepted 24 August 2011 - First published online 9 December 2011)
}

\begin{abstract}
Inflammatory bowel disease (IBD) is a collective term for conditions characterised by chronic inflammation of the gastrointestinal tract involving an inappropriate immune response to commensal micro-organisms in a genetically susceptible host. Previously, aqueous and ethyl acetate extracts of gold kiwifruit (Actinidia chinensis) or green kiwifruit (A. deliciosa) have demonstrated anti-inflammatory activity using in vitro models of IBD. The present study examined whether these kiwifruit extracts (KFE) had immune-modulating effects in vivo against inflammatory processes that are known to be increased in patients with IBD. KFE were used as a dietary intervention in IL-10-genedeficient $\left(I l 10^{-1-}\right.$ ) mice (an in vivo model of IBD) and the $\mathrm{C} 57 \mathrm{BL} / 6 \mathrm{~J}$ background strain in a $3 \times 2$ factorial design. While all $I l 10^{-1-}$ mice developed significant colonic inflammation compared with $\mathrm{C} 57 \mathrm{BL} / 6 \mathrm{~J}$ mice, this was not affected by the inclusion of KFE in the diet. These findings are in direct contrast to our previous study where KFE reduced inflammatory signalling in primary cells isolated from $I l 10^{-/-}$and C57BL/6J mice. Whole-genome gene and protein expression level profiling indicated that KFE influenced immune signalling pathways and metabolic processes within the colonic tissue; however, the effects were subtle. In particular, expression levels across gene sets related to adaptive immune pathways were significantly reduced using three of the four KFE in C57BL/6J mice. The present study highlights the importance of investigating food components identified by cell-based assays with appropriate in vivo models before making dietary recommendations, as a food that looks promising in vitro may not be effective in vivo.
\end{abstract}

Key words: Inflammatory bowel disease: Kiwifruit extract: Gene expression: Proteomics

Inflammatory bowel disease (IBD) is a term describing chronic inflammatory conditions of the gastrointestinal tract consisting of two main subtypes, Crohn's disease and ulcerative colitis, with differing pathology and immunopathogenesis ${ }^{(1)}$. The pathogenesis of IBD is complex, involving both genetic and environmental components that may differ among patients. While diet is thought to be an important factor in IBD, there is little evidence at present for the involvement of specific food components in either aetiology or treatment. This may be because of variability in the response to food components among IBD patients, where each patient tolerates, or is sensitive to, a range of foods such that no single food is associated with all patients ${ }^{(2,3)}$.

Kiwifruit, the fruit of the Actinidia genus, contain a number of nutritionally important compounds, including vitamin C, folate, $\mathrm{K}, \mathrm{Mg}$ and fibre ${ }^{(4,5)}$, as well as many plant secondary compounds such as carotenoids, polyphenols and terpenoids ${ }^{(6-8)}$. Several health benefits have been demonstrated for kiwifruit, including protection against carcinogenesis ${ }^{(9)}$, protection against oxidative stress and DNA damage ${ }^{(10-12)}$, enhanced adaptive immune response ${ }^{(13,14)}$ and improved laxity $^{(15,16)}$. In addition, extracts from gold kiwifruit (Actinidia

Abbreviations: DIGE, differential in-gel electrophoresis; FC, fold change; GSEA, gene set enrichment analysis; HIS, histological injury score; IBD, inflammatory bowel disease; $I l 10^{-1-}$, IL-10 gene deficient; KFE, kiwifruit extract; MAPK, mitogen-activated protein kinase; qRT-PCR, quantitative RT-PCR; TLR, Toll-like receptor.

*Corresponding author: Dr W. A. Laing, fax +64 99258628, email william.laing@plantandfood.co.nz 
chinensis 'Hort16A') and green kiwifruit (A. deliciosa 'Hayward') have been reported to suppress Toll-like receptor (TLR) signalling by innate immune cells in vitro, reducing the secretion of pro-inflammatory mediators such as NO or cytokines after cellular activation by bacterial antigens ${ }^{(12,17-19)}$.

The IL-10-gene deficient ( Il1O $^{-/-}$) mouse develops Crohn's disease-like colitis when exposed to commensal microbiota and is extensively used as a model for $\operatorname{IBD}^{(20,21)}$. Inflammation develops in discontinuous, transmural lesions along the length of the intestine, with infiltration of the lamina propria by large numbers of activated macrophages and increased differentiation of Th1 and Th17 cells ${ }^{(20,22-24)}$. In addition, the molecular changes within the inflamed colon of the $111 \mathrm{O}^{-/-}$mouse have been characterised ${ }^{(25-27)}$.

In a previous paper, we used primary cells isolated from IllO ${ }^{-1-}$ and the $\mathrm{C} 57 \mathrm{BL} / 6 \mathrm{~J}$ background strain to test the in vitro activity of kiwifruit extracts $(\mathrm{KFE})^{(19)}$. Anti-inflammatory activity was observed against TLR-driven activation of both macrophages and intestinal epithelial cells, which is a cellular process known to play a key role in the development of colitis in IBD patients ${ }^{(19,22,28)}$. Significant activity was observed in cells isolated from $I l 1 O^{-/-}$as well as wild-type mice $^{(19)}$, suggesting that IL-10 is not required for KFE antiinflammatory activity. Cell-based assays play an important role in nutrition research, as they allow the rapid identification of potentially beneficial food components from a very large pool of candidates ${ }^{(29)}$; however, further in vivo testing is necessary to investigate whether beneficial activity persists in the whole animal. Given the positive in vitro results, we progressed to the $1 l 10^{-/-}$mouse as a suitable in vivo model for investigating KFE anti-inflammatory activity, particularly with regard to the genes and pathways involved in the chronic inflammation of IBD. Our hypothesis was that consumption of diets containing KFE would suppress cellular activation in vivo, leading to a reduction in colitis and immune signalling. Therefore, we investigated the effects of KFE consumption by $I l 1 \mathrm{O}^{-/-}$and $\mathrm{C} 57 \mathrm{BL} / 6 \mathrm{~J}$ background strain mice on weight gain, colonic inflammation, and colonic gene and protein expression levels.

\section{Methods and materials}

The study was reviewed and approved by the AgResearch Ruakura Animal Ethics Committee, Hamilton, New Zealand according to the New Zealand Animal Welfare Act 1999.

\section{Animals and diets}

A total of seventy-five male $1110^{-/-}$mice (B6-129P2.Il10 $<\operatorname{tm} 1 \mathrm{Cgn}>/ \mathrm{J}$ ) and forty-four $\mathrm{C} 57 \mathrm{BL} / 6 \mathrm{~J}$ control mice were purchased from The Jackson Laboratories (Bar Harbor, ME, USA) at 4-6 weeks of age. Mice were housed singly in shoebox-style cages $(332 \times 150 \times 130 \mathrm{~mm})$ containing Alpha-Dri litter (Shepherd Specialty Papers Inc., Kalamazoo, MI, USA) and a plastic tube for environmental enrichment. The animals were maintained in a temperature- and humidity-controlled room with a $12 \mathrm{~h}$ light $-12 \mathrm{~h}$ dark cycle.

KFE were prepared as described previously ${ }^{(19)}$, and incorporated into powdered AIN-76A diets prepared in-house following the standard recipe ${ }^{(30,31)}$. A proportion of the sugar was replaced with appropriate amounts of $\mathrm{KFE}$, as shown in Table 1. All diets used in the present study were shown to be palatable and non-toxic to $\mathrm{C} 57 \mathrm{BL} / 6 \mathrm{~J}$ mice under these experimental conditions (SJ Edmonds, unpublished results).

The following experiments were conducted: Expt 1 tested diets supplemented with gold KFE and Expt 2 tested diets supplemented with green KFE (Table 1 ). Before each experimental period, $I l 10^{-1-}$ and $\mathrm{C} 57 \mathrm{BL} / 6 \mathrm{~J}$ mice were assigned to treatment groups in a randomised block design. After a $3 \mathrm{~d}$ acclimatisation period, all mice were inoculated with a combination of twelve strains of Enterococcus faecium or E. faecalis and intestinal flora derived from $\mathrm{C} 57 \mathrm{BL} / 6$ mice raised under conventional conditions, as described previously ${ }^{(25,26)}$

Mice were offered fresh food daily and the average food intake was estimated by the collection and weighing of uneaten food. Leftover food was removed from the feeder and the bedding was strained using a standard kitchen sieve to collect any waste food scattered throughout the cage. This ensured the collection and measurement of all uneaten food allowing

Table 1. Treatment groups and mouse numbers

Kiwifruit extract added to diet

\begin{tabular}{|c|c|c|c|c|c|}
\hline Expt & Genotype & Treatment & Type & \%Diet & No. of mice \\
\hline \multirow[t]{6}{*}{1} & C57BL/6J & Gold control & None & & 8 \\
\hline & & Gold aqueous & Gold aqueous & $5 \cdot 0$ & 8 \\
\hline & & Go ethyl acetate & Gold ethyl acetate & 0.11 & 8 \\
\hline & $1 / 10^{-1-}$ & Gold control & None & & 15 \\
\hline & & Gold aqueous & Gold aqueous & $5 \cdot 0$ & 15 \\
\hline & & Go ethyl acetate & Gold ethyl acetate & 0.11 & 15 \\
\hline \multirow[t]{6}{*}{2} & C57BL/6J & Green control & None & & 7 \\
\hline & & Green aqueous & Green aqueous & $5 \cdot 0$ & 6 \\
\hline & & Green ethyl acetate & Green ethyl acetate & 0.11 & 7 \\
\hline & $1110^{-1-}$ & Green control & None & & 10 \\
\hline & & Green aqueous & Green aqueous & 5.0 & 10 \\
\hline & & Green ethyl acetate & Green ethyl acetate & 0.11 & 10 \\
\hline
\end{tabular}

$\%$ Diet, percentage of diet.

* The base diet consisted of AIN-76A prepared in-house following the standard recipe ${ }^{(30,31)}$. Kiwifruit extracts were prepared as described previously ${ }^{(19)}$. 
consistent estimation of food intake regardless of animal activity. Food was supplied ad libitum for the first $20 \mathrm{~d}$, and then for the remainder of the experimental period, the food offered was adjusted to equal the mean amount of food consumed by $I l 10^{-/-}$mice fed plain AIN-76A during the previous week. Water was provided ad libitum. Mice were weighed three times per week to determine body-weight changes, and their overall condition assessed and a general health score ${ }^{(32)}$ determined $6 \mathrm{~d} /$ week.

\section{Tissue sampling}

A final body-weight measurement was taken $41 \mathrm{~d}$ after inoculation. Tissue sampling was carried out on days 42-44. Before euthanasia, mice were fasted overnight for $14 \mathrm{~h}$, fed for $2 \mathrm{~h}$, and then fasted again for $2 \mathrm{~h}$ to reduce variation in timing of the last food intake for each animal before tissue collection ${ }^{(33)}$. Animals were euthanised by $\mathrm{CO}_{2}$ asphyxiation followed by cervical dislocation. Blood was collected by cardiac puncture $(0.5-1 \mathrm{ml})$, anticoagulated with $0.5 \mathrm{~m}$-EDTA (Invitrogen, Carlsbad, CA, USA); the plasma was separated by centrifugation $\left(4 \mathrm{~min}, 3000 \mathrm{~g}, 4^{\circ} \mathrm{C}\right.$ ), frozen in liquid $\mathrm{N}_{2}$ and stored at $-80^{\circ} \mathrm{C}$ for cytokine analysis.

The gastrointestinal tract was removed, cut open lengthwise and flushed with $0.9 \% \mathrm{NaCl}$ to remove any traces of digesta. Sections of the colon were rapidly frozen in liquid $\mathrm{N}_{2}$ before storage at $-80^{\circ} \mathrm{C}$. A subsample from each colon was fixed in $10 \%$ phosphate-buffered formalin immediately after dissection and stored at room temperature until histological evaluation.

\section{Histology}

The formalin-fixed samples from each colon were embedded in a paraffin block, cut into $5 \mu \mathrm{m}$ sections and then stained with haematoxylin and eosin for light microscopic examination. Each tissue was scored for the aspects of inflammation related to inflammatory lesions, tissue destruction or tissue reparation, and a total histological injury score (HIS) was calculated as described previously ${ }^{(25)}$. The total HIS of intestinal sections collected from $1110^{-1-}$ mice has been shown to correlate with validated measures of intestinal inflammation, thus providing a quantitative measure of colitis ${ }^{(34)}$

\section{Plasma IL-6}

IL-6 levels in plasma were determined as a biomarker of inflammation using Ready-Set-Go! ${ }^{\circledR}$ pre-coated mouse IL6 ELISA plates (88-7964-29; eBioscience, San Diego, CA, USA), following the manufacturer's protocol.

\section{Statistical analysis}

Statistical analyses of body-weight change, food intake, colon HIS and plasma IL-6 concentration were performed in GenStat (Tenth Edition; VSN International, Hemel Hempstead, UK, 2005). All results are expressed as means with their standard errors of the mean. Mouse weight gain and average daily food intake were assessed using two-way ANOVA. The initial weight of the mouse was used as a covariate for weight-gain data. Diet, strain and interaction means were obtained for the average values of each parameter being tested, and were compared using the appropriate least significant difference (at the 5\% significance level) between means.

The effects of the diet on average colon HIS or plasma IL-6 concentration were assessed for $\mathrm{IlIO}^{-/-}$mice using one-way ANOVA based on log-transformed values. Within-diet means were obtained and compared using the appropriate least significant difference (at the 5\% significance level) between means. C57BL/6J mice were not analysed for these measures because of the high proportion of zero values across all dietary treatment groups.

\section{mRNA preparation}

mRNA was isolated from each colon tissue sample using the standard TRIzol protocol (Invitrogen). The extracted mRNA was dissolved in $20 \mu \mathrm{l}$ RNase-free water and then purified using the Qiagen RNeasy Mini Kit (Qiagen, San Diego, CA, USA). Reference RNA was prepared from equal amounts of total purified RNA extracted from several organ tissues (small intestine, colon, kidney, liver and fetuses) of healthy Swiss mice to include transcripts for most of the probes that are present on the array. mRNA concentration and purity (A260:A280 ratio) were determined using a Nanodrop ND-1000 spectrophotometer (Nanodrop Technologies, Wilmington, DE, USA) and overall RNA quality was assessed using an Agilent 2100 Bioanalyser (RNA 6000 Nanochip; Agilent Technologies, Santa Clara, CA, USA). Only mRNA with an A260:A280 ratio $>2.0$ and Bioanalyser RNA integrity number $>8.0$ was used for microarray hybridisation or quantitative RT-PCR (qRT-PCR) analysis.

\section{Microarrays}

RNA from samples and the reference pool was amplified and labelled using Agilent's Low RNA Input Linear Amplification Kit PLUS (Agilent Technologies), according to the manufacturer's instructions. Briefly, $500 \mathrm{ng}$ of purified total RNA from each sample were reverse transcribed into complementary DNA using T7 RNA polymerase, which was subsequently labelled with either cyanine 3-CTP (sample) or cyanine 5-CTP (reference) dyes (10 mm; Perkin-Elmer/NEN Life Science, Boston, MA, USA). The fluorescently labelled cRNA was hybridised onto Agilent Technologies Whole Mouse Genome 60 mer Oligo $4 \times 44 \mathrm{~K}$ microarrays using the Agilent Gene Expression Hybridization Kit in accordance with the manufacturer's instructions. A reference design (without dye swap) was used whereby one sample and a common reference were hybridised on to each two-colour array.

Hybridised arrays were scanned using an Agilent microarray scanner and the resulting data with Agilent feature extraction software version 9.5.1 (Agilent Technologies). The microarray data are available as accession GSE27684 in the Gene Expression Omnibus repository at the National Center for 
Biotechnology Information (http://www.ncbi.nlm.nih.gov/ geo/info/linking.html).

Data preprocessing and analysis of differential expression were conducted using Bioconductor ${ }^{(35)}$ under $\mathrm{R}$ 2.9.2. The quality of the microarray data was assessed by diagnostic plots (box plots and density plots), and spatial images were generated using the arrayQuality (version 1.24.0) and arrayQualityMetrics (version 2.4.3) packages from Bioconductor. Data were normalised within each array using local polynomial regression fitting normalisation, and then between arrays using quantile normalisation of the red channel containing the common reference RNA sample ${ }^{(36)}$, as described previously ${ }^{(37)}$. Background subtraction was unnecessary because of homogeneous hybridisation.

Differentially expressed genes were identified using the limma (version 3.2.2) package (http://www.bioconductor. org/packages/2.8/bioc/html/limma.html $)^{(38,39)}$ and cut-off thresholds of adjusted $P$ value $\leq 0.05$ and fold change (FC) $\geq|1.5|$ were used to determine significance.

Gene set enrichment analysis (GSEA) was conducted using the GSEA-P Java Application version 2.0.5 (http://www. broadinstitute.org/gsea/ $)^{(40)}$ to identify functionally related groups of genes (gene sets) that have statistically significant, concordant differences between two biological states ${ }^{(41,42)}$. All gene sets tested were downloaded from the MSigDB database version 2.5 (http://www.broadinstitute.org/gsea/msigdb/ index.jsp) ${ }^{(40)}$. Because of the low replicate numbers within each treatment group $(n<7)$, gene_set permutations were used and gene sets were considered significantly enriched when the false discovery rate $q$ value was $\leq 0.05$ and Fisher's exact test nominal $P$ value was $\leq 0.01$, as suggested in the GSEA-P user instructions.

\section{Quantitative RT-PCR}

The following genes were selected for validation: matrix metallopeptidase 13 (Mmp13); matrix metallopeptidase 10 (Mmp10); S100 calcium-binding protein A8 (S100a8); defensin, alpha, 21 (Defa21); sulfotransferase family 1D, member 1 (Sult1d1); regenerating islet-derived 3 beta (Reg3b); mitogen-activated protein kinase 13 (Mapk13); insulin-like growth factor binding protein 5 (Igfbp5) and fatty acid-binding protein 2 (Fabp2). Expression levels of these genes were established using qRT-PCR. Complementary DNA was synthesised from the same total mRNA samples used for the microarray analysis using the SuperScript VILO cDNA Synthesis Kit (Invitrogen). Reverse transcription was performed using $0.9 \mu \mathrm{g}$ total RNA and oligo-dT primers, according to the manufacturer's instructions.

Data were normalised against three reference genes, calnexin (Canx), MON2 homologue (yeast) (Mon2) and mitogen-activated protein kinase kinase 1 (Map2k1), using the method described by Vandesompele et $a l .{ }^{(43)}$. Expression levels of these genes were stable between the treatment groups when assessed by microarray analysis and qRT-PCR. Primers for Canx were designed using PrimerSelect software (DNASTAR Lasergene, Madison, WI, USA), as described previously ${ }^{(44)}$. Primers for the remaining genes were designed using Primer 3.0 software (http://primer3.sourceforge.net/) ${ }^{(45)}$ and evaluated using the RTPrimerDB in silico assay evaluation to avoid primer secondary structures ${ }^{(46)}$. Primer sequences for reference or target genes are shown in Table 2 . The specificities of all PCR were verified by melting curve analysis and agarose gel electrophoresis.

The PCR conditions were as follows: $95^{\circ} \mathrm{C}$ for $5 \mathrm{~min}$, forty-five cycles at $95^{\circ} \mathrm{C}$ for $15 \mathrm{~s}, 60^{\circ} \mathrm{C}$ for $10 \mathrm{~s}$ and $72^{\circ} \mathrm{C}$ for $15 \mathrm{~s}$. Melting curve analyses were performed by increasing the temperature $\left(1^{\circ} \mathrm{C} / \mathrm{s}\right)$ from 65 to $95^{\circ} \mathrm{C}$, with continuous fluorescence acquisition. Threshold cycle $\left(C_{t}\right)$ values were obtained in quadruplicate for each sample using a LightCycler 480 (Roche Diagnostics, Auckland, New Zealand) and LightCycler 480 SYBR Green I Master (Roche Diagnostics) in $10 \mu \mathrm{l}$ reactions, according to the manufacturer's protocol. LightCycler 480 Relative Quantification Software (Roche, Auckland, New Zealand) was used to calculate mRNA concentration and normalised ratios (target:reference) based on standard curves generated using serial dilutions of pooled complementary DNA from all samples.

\section{Protein preparation}

Protein pellets were extracted from the same colon samples as mRNA using the combined TRIzol extraction, according to the manufacturer's protocol. The protein pellets were precipitated with $3 \mathrm{ml}$ isopropanol, washed five times with $0.3 \mathrm{M}$-guanidine hydrochloride (Invitrogen) in 95\% ethanol, and then

Table 2. Quantitative RT-PCR genes and primers

\begin{tabular}{|c|c|c|c|c|c|}
\hline Gene type & Agilent probe ID & Accession no.* & Gene symbol & Forward primer $\left(5^{\prime}-3^{\prime}\right)$ & Reverse primer $\left(5^{\prime}-3^{\prime}\right)$ \\
\hline Target & A_51_P184484 & NM_008607.2 & Mmp13 & CAGTGGAGGTGGCCTTACAT & GAAATCTCCTCCATTTCTCTCTCA \\
\hline Target & A_51_P120830 & NM_019471.2 & Mmp10 & CCAGGACGGTGACACACATA & CACAGAACATGCAGGAGCAA \\
\hline Target & A_51_P256827 & NM_013650.2 & S100a8 & GGAAATCACCATGCCCTCTA & ATCACCATCGCAAGGAACTC \\
\hline Target & A_51_P460391 & NM_183253.3 & Defa21 & CCAGGCTGTGTCTGTCTCCT & GCGCAGGTCCCATAAAATAG \\
\hline Target & A_51_P481721 & NM_016771.3 & Sult1d1 & ААTTATCTTCCTTACAGAAAGGTTCA & TTCCTCTAGGAGGCCACTGA \\
\hline Target & A_51_P169671 & NM_011036.1 & $\operatorname{Reg} 3 b$ & GCAAACATCCCGAATTTGTC & GCCCAAACTTATACCAAAAGGA \\
\hline Target & A_51_P239203 & NM_011950.2 & Mapk13 & CATAGCCCGGAAGGACTCAC & GAGGTGGGTGGATCTCTTGA \\
\hline Target & A_51_P204153 & NM_010518.2 & $\operatorname{lgfbp5}$ & TTGCCTCAACGAAAAGAGCTAC & CACAGTTGGGCAGGTACACAG \\
\hline Target & A_52_P453013 & NM_007980.2 & Fabp2 & GTGGAAAGTAGACCGGAACGA & CCATCCTGTGTGATTGTCAGTT \\
\hline Reference & A_51_P242143 & NM_007597.2 & Canx & CTGAAGGCTGGCTAGACGACGAA & GCTGACTCACACTTGGGGTTGG \\
\hline Reference & A_51_P401458 & NM_153395.2 & Mon2 & TGCTTCACACCTGCTACCAT & AAAAGGGTGCAAAACACCAG \\
\hline Reference & A_51_P241074 & NM_008927.3 & Map2k1 & AGTGGATTGGCTTTGTGCTT & TACAGGCAGCCAGCTAGTGA \\
\hline
\end{tabular}

* National Center for Biotechnology Information Entrez Gene (http://www.ncbi.nlm.nih.gov/sites/entrez?db=gene). 
washed once in $100 \%$ ethanol (BDH Absolute; Biolab Limited, Auckland, New Zealand) and allowed to dry. Each sample was solubilised as described previously ${ }^{(27)}$, and an aliquot of each sample was purified using the Amersham Biosciences 2-D Clean-Up Kit (GE Healthcare, Auckland, New Zealand), according to the manufacturer's instructions. The resulting pellet was resolubilised as described previously ${ }^{(27)}$, centrifuged briefly to remove insoluble protein, and the protein content of the supernatant determined using the Bio-Rad Protein Assay (BioRad, Gladesville, Australia) based on the Bradford reagent $^{(47)}$

\section{Two-dimensional gel electrophoresis}

Two-dimensional gel electrophoresis was undertaken according to a modified version of a previously described proto$\mathrm{Col}^{(48)}$. According to this protocol, six biological replicates for each comparison were analysed using two gels, where each gel contained pooled samples from three individual mice within the same treatment group (16.67 $\mu \mathrm{g}$ protein/ mouse, $50 \mu \mathrm{g}$ protein total). Pooling was necessary to reduce individual noise between mice and increase the amount of protein available for analysis within each comparison.

Treatment and control sample pools were labelled with 200 pmol cyanine-2 and cyanine-5 dyes (GE Healthcare, Uppsala, Sweden), respectively, as described by the manufacturer. The labelled pools for each gel were combined to give $100 \mu \mathrm{g}$ protein, and then prepared, loaded and isoelectrically focused on commercially available precast immobilised $\mathrm{pH}$ gradient (IPG) strips $(18 \mathrm{~cm})$ with a non-linear $\mathrm{pH}$ 3-11 gradient, as described previously ${ }^{(27,48)}$. IPG strips were equilibrated and proteins separated in the second dimension by SDS-PAGE using vertical 10\% SDS-PAGE gels $(200 \times 160 \times 1.5 \mathrm{~mm})$, as described previously ${ }^{(27,48)}$. Precision Plus protein standard plugs (Bio-Rad Laboratories, Auckland, New Zealand) were used as molecular weight markers.

Immediately after electrophoresis, the gels were rinsed in reverse osmosis water and then scanned using a Typhoon 9400 imager (Amersham BioSciences, GE Healthcare). Scan settings were as follows: $100 \mu \mathrm{m}$ resolution; differential in-gel electrophoresis (DIGE) file naming format selected; Cy5 scanned using a $488 \mathrm{~nm}$ laser and a $520 \mathrm{~nm}$ bandpass $40 \mathrm{~nm}$ emission filter, PMT $520 \mathrm{~V}$; Cy2 scanned using a $633 \mathrm{~nm}$ laser and a $670 \mathrm{~nm}$ bandpass $30 \mathrm{~nm}$ emission filter, PMT 490 V. Spot patterns between gel images were analysed using Shimadzu 2D Evolution version 2005 software (Nonlinear Dynamics Limited, Newcastle upon Tyne, UK) to find differentially expressed spots between samples within each gel. Differential expression of a spot was considered to be significant where the abundance FC for each biological replicate changed within the same direction, and $|F C|$ was $\geq 2.0$ in one replicate and $\geq 1.3$ in the second replicate in the same direction

Once the gel image was captured, each gel was stained with Sypro Ruby (Invitrogen), followed by overstaining with Colloidal Coomassie Blue stain, as described previously ${ }^{(48)}$, to visualise spots for later removal and identification. Gels were dried between cellophane layers on glass plates at room temperature for long-term storage.

\section{Protein spot identification}

The spots corresponding to each protein of interest were located visually on each gel and one replicate was chosen for identification. Each of the chosen spots was excised using a razor blade and placed into an individual $1.5 \mathrm{ml} \mathrm{micro-}$ centrifuge tube. A similarly sized piece of gel was excised from a protein-free region of the gel to identify trypsin autoproteolysis products. All gel pieces were rehydrated in deionised water and digested with trypsin, as described previously ${ }^{(27)}$. The resulting tryptic peptides were separated and analysed using an Ettan multidimensional liquid chromatography system (GE Healthcare) coupled to an linear trap quadrupole (LTQ) linear ion trap mass spectrometer with a nanospray ionisation interface (ThermoQuest, San Jose, CA, USA), as described previously ${ }^{(27)}$.

\section{Results}

\section{Food intake and body weight}

During the early stages of the experimental period, only one IllO ${ }^{-/-}$mouse in Expt 1 developed an infected eye. As this may have influenced the overall inflammatory state and general health of this animal, it was withdrawn from all subsequent analysis. This left a final group size of fourteen for the $I l 1 O^{-/-}$aqueous gold KFE group.

Due to issues with animal supply, the average body weights of mice at the start of the experimental period were significantly different between genotypes for Expt 1, but not for Expt 2, despite all animals within each experiment being the same age at delivery. After randomisation, the average initial body weights of mice assigned to each dietary group were not significantly different within genotypes (Table 3).

A covariate analysis found that initial mouse weight had a significant effect on weight gain in Expt 1 (coefficient $=$ $-0.33, P<0.001$ ) but not in Expt 2 (coefficient $=5.99$, $P=0.08)$, with a negative coefficient indicating that, in general, the higher the initial weight, the smaller the total weight gain. However, the body-weight gain was significantly lower for $I l 1 O^{-/-}$mice than for age-matched $\mathrm{C} 57 \mathrm{BL} / 6 \mathrm{~J}$ mice in both experiments $(P<0.001)$ regardless of covariate adjustment, indicating a reduced growth rate for $1 l 10^{-/-}$mice. This was accompanied by a deterioration in health and overall condition of $1 l 10^{-/-}$, but not $\mathrm{C} 57 \mathrm{BL} / 6 \mathrm{~J}$, mice by the end of the experimental period.

There were no differences in general health score, overall condition or total weight gain when comparing each KFE treatment diet with the appropriate control diet within each genotype (Table 2). However, there were significant differences between genotypes within each diet, with $\mathrm{C} 57 \mathrm{BL} / 6 \mathrm{~J}$ mice gaining more weight than $1 l 10^{-/-}$mice fed the same diet. This was confirmed by two-way ANOVA for each experiment, which detected a significant difference between genotypes, but not between diets, and no diet $\times$ genotype interaction. 
Table 3. Animal characteristics per genotype and diet for Expt 1 and Expt 2

\begin{tabular}{|c|c|c|c|c|c|c|c|c|c|c|c|}
\hline \multirow[b]{2}{*}{ Variables } & \multicolumn{2}{|c|}{$\begin{array}{l}\text { Control } \\
\text { diet }\end{array}$} & \multicolumn{2}{|c|}{$\begin{array}{l}\text { Aqueous } \\
\text { KFE }\end{array}$} & \multicolumn{2}{|c|}{$\begin{array}{c}\text { Ethyl acetate } \\
\text { KFE }\end{array}$} & \multirow[b]{2}{*}{ SED } & \multirow[b]{2}{*}{ df } & \multicolumn{3}{|c|}{$P^{*}$} \\
\hline & $\mathrm{C} 57$ & $1 / 10^{-1-}$ & $\mathrm{C} 57$ & $1110^{-1-}$ & $\mathrm{C} 57$ & $1110^{-1-}$ & & & Genotype & Diet & Interaction \\
\hline \multicolumn{12}{|l|}{ Gold KFE experiment } \\
\hline Mice $(n)$ & 8 & 15 & 8 & 14 & 8 & 15 & & & & & \\
\hline Initial body weight† (g) & $15 \cdot 6$ & $16 \cdot 0$ & $15 \cdot 9$ & $15 \cdot 7$ & $15 \cdot 2$ & $16 \cdot 1$ & 0.9 & 63 & 0.46 & 0.99 & 0.69 \\
\hline Unadjusted weight gain (g) & $7 \cdot 6$ & $6 \cdot 7$ & 7.9 & $6 \cdot 7$ & $8 \cdot 4$ & $7 \cdot 0$ & 0.6 & 62 & $<0.001$ & 0.37 & 0.85 \\
\hline Adjusted weight gain $\ddagger$ (g) & 7.5 & $6 \cdot 7$ & 7.9 & $6 \cdot 7$ & $8 \cdot 2$ & $7 \cdot 1$ & 0.5 & 61 & $<0.001$ & 0.29 & 0.82 \\
\hline Food intake (mean $\mathrm{g} / \mathrm{d}$ ) & 3.5 & 3.4 & 3.5 & 3.6 & 3.5 & 3.5 & 0.1 & 62 & 0.51 & 0.60 & 0.59 \\
\hline Final GHS $\leq 4(n)$ & 0 & 5 & 0 & 3 & 0 & 7 & & & & & \\
\hline Final GHS $=5(n)$ & 8 & 10 & 8 & 11 & 8 & 8 & & & & & \\
\hline Colon HIS & 0.3 & 6.7 & 0.5 & $7 \cdot 3$ & 0.6 & 6.4 & 0.5 & 62 & $<0.001$ & $0.35 \S$ & $\mathrm{N} / \mathrm{A} \|$ \\
\hline Plasma IL-69 & 65 & 960 & 25 & 1120 & 0.0 & 783 & 413 & 59 & $<0.001$ & $0.71 \S$ & N/A\| \\
\hline \multicolumn{12}{|l|}{ Green KFE experiment } \\
\hline Mice $(n)$ & 7 & 10 & 6 & 10 & 7 & 10 & & & & & \\
\hline Initial body weight† (g) & 19.5 & $19 \cdot 4$ & $19 \cdot 7$ & $19 \cdot 6$ & $19 \cdot 7$ & $19 \cdot 4$ & 1.0 & 44 & 0.95 & 1.00 & 0.90 \\
\hline Unadjusted weight gain (g) & 7.9 & 4.5 & 7.8 & 4.3 & 7.9 & 4.6 & 0.8 & 44 & $<0.001$ & 0.60 & 0.99 \\
\hline Adjusted weight gain $¥$ (g) & 7.5 & 4.5 & $7 \cdot 2$ & 4.3 & $7 \cdot 7$ & 4.8 & 0.7 & 43 & $<0.001$ & 0.59 & 0.99 \\
\hline Food intake (mean $\mathrm{g} / \mathrm{d})$ & 3.0 & 2.9 & $3 \cdot 2$ & $3 \cdot 0$ & 3.2 & 2.9 & 0.1 & 44 & $<0.001$ & 0.52 & 0.55 \\
\hline Final GHS $\leq 4(n)$ & 0 & 6 & 0 & 9 & 0 & 6 & & & & & \\
\hline Final GHS $=5(n)$ & 6 & 4 & 7 & 1 & 7 & 4 & & & & & \\
\hline Colon HIS & 0.5 & $7 \cdot 6$ & 0.7 & $7 \cdot 2$ & 0.6 & $7 \cdot 0$ & 0.4 & 44 & $<0.001$ & $0.69 \S$ & $N / A \|$ \\
\hline Plasma IL-6ฮ & 0.0 & 744 & 40 & 549 & 38 & 780 & 338 & 40 & 0.002 & $0.83 \S$ & N/A\| \\
\hline
\end{tabular}

KFE, kiwifruit extract; SED, average standard error of difference between two means; df, residual degrees of freedom for the test of significance of each term; GHS, general health score ${ }^{(32)} ;$ HIS, histology injury score s. $^{(25)}$

* Values were significantly different $(P<0.05)$.

$\uparrow$ Mouse body weight on day 1 of the experimental period.

$\ddagger$ Covariate = body weight on day 1 .

$\S$ One-way ANOVA using data from $1 / 10^{-/-}$mice only.

|| Interaction not measured because of the large number of zero values in the C57BL/6J data.

If Expressed as $\mathrm{ng} \mathrm{IL-6/ml}$ plasma per mg final mouse body weight.

Therefore, the KFE-supplemented diet had no effect on food intake, animal weight gain or overall animal health.

In Expt 1, there were no significant differences in food intake between genotypes or diets, and no diet $\times$ genotype interaction. However, in Expt 2, the daily food intake of $I l 10^{-/-}$mice dropped after day 20. Therefore, the amount of food offered to $\mathrm{C} 57 \mathrm{BL} / 6 \mathrm{~J}$ mice in this experiment was reduced, with the aim of preventing $\mathrm{C} 57 \mathrm{BL} / 6 \mathrm{~J}$ mice from consuming more food than their $\mathrm{IllO}^{-/-}$counterparts. Twoway ANOVA assessing average daily food intake indicated that this was not successful, with a significant difference in food intake between genotypes, but not diets, detected for this experiment (Table 3).

\section{Colonic and systemic inflammation}

Histological sections from colon samples were examined and a colon HIS was assigned to each animal (Table 3). A colon HIS was unable to be assigned to one $1110^{-/-}$mouse in the ethyl acetate gold KFE dietary group because of incorrect sampling, where tissue was taken from the wrong part of the colon. Therefore, histology data from this animal were omitted from further analysis. There was no colonic inflammation present in $\mathrm{C} 57 \mathrm{BL} / 6 \mathrm{~J}$ mice, with all HIS being 1.0 or below. In contrast, all $1110^{-1-}$ mice displayed medium to high inflammation, with HIS values ranging between 3.5 and $11 \cdot 5$. However, there were no significant differences in colon HIS between diets within $\mathrm{Il}_{10}^{-/-}$mice.
The absence of any effect of KFE on colon inflammation in Il1O ${ }^{-1-}$ mice was supported by plasma IL-6 concentrations (Table 3). Plasma IL-6 was significantly increased in $1 l 10^{-1-}$ mice compared with $\mathrm{C} 57 \mathrm{Bl} / 6 \mathrm{~J}$ mice $(P<0.001)$, indicating a significant increase in systemic inflammation; however, there were no significant dietary effects and or diet $\times$ genotype interactions in either experiment.

\section{Changes in colonic gene expression levels}

Box plots of the $\log 2$ (intensities) generated by the array quality metrics package indicated quality issues with two arrays, one from each experiment, and they were removed from all further statistical analysis. The remaining seventy arrays passed quality inspection and were analysed.

LIMMA analysis of colonic gene expression levels detected no significantly differentially expressed genes for any KFE-supplemented diet when compared with the control diet within each genotype. GSEA assessment detected a total of 159 significantly enriched gene sets, with between two and sixty-four sets identified within each comparison (Table 4)

GSEA results for gold and green aqueous KFE were similar (Table 4). Expression levels across gene sets related to T-cell activation and adaptive immunity were increased in the colon samples from $\mathrm{C} 57 \mathrm{Bl} / 6 \mathrm{~J}$ mice fed these extracts when compared with those fed the control diet, while expression levels across gene sets related to carbohydrate and energy 
Table 4. Gene set enrichment analysis (GSEA) of pathways up- or down-regulated in the mouse colon by the kiwifruit extract (KFE)-supplemented $\operatorname{diets}^{*}$

\begin{tabular}{|c|c|c|c|c|c|c|}
\hline MSigDB gene set name $†$ & Size & ES & NES $\ddagger$ & FDR $q$-value§ & FE $P \S$ & Function \\
\hline \multicolumn{7}{|l|}{ Aqueous gold KFE } \\
\hline \multicolumn{7}{|l|}{ C57BL/6J } \\
\hline Positive regulation of T-cell activation\| & 20 & 0.62 & 1.93 & 0.041 & 0.002 & Immune/inflammation \\
\hline Complement and coagulation cascades & 52 & 0.51 & 1.99 & 0.042 & $<0.001$ & Immune/inflammation \\
\hline Positive regulation of cytokine biosynthetic process $\|$ & 24 & 0.62 & 1.99 & 0.026 & $<0.001$ & Immune/inflammation \\
\hline $\begin{array}{l}\text { Positive regulation of translation } \| \\
\| 110^{-1-}\end{array}$ & 31 & 0.65 & $2 \cdot 21$ & 0.000 & $<0.001$ & Transcription/translation \\
\hline Pyrimidine metabolism\| & 55 & -0.62 & $-2 \cdot 18$ & 0.000 & $<0.001$ & Nucleoside metabolism \\
\hline Pyrimidine metabolism & 82 & -0.56 & $-2 \cdot 15$ & 0.000 & $<0.001$ & Nucleoside metabolism \\
\hline Citrate cycleq & 23 & 0.65 & $2 \cdot 21$ & 0.007 & $<0.001$ & Carbohydrate metabolism \\
\hline Krebs/TCA cycle ${ }^{\star \star}$ & 27 & 0.67 & $2 \cdot 32$ & 0.004 & $<0.001$ & Carbohydrate metabolism \\
\hline Lipid transport\| & 24 & 0.56 & 1.88 & 0.042 & $<0.001$ & Lipid transport \\
\hline Biopolymer catabolic process $\|$ & 96 & 0.42 & 1.88 & 0.038 & $<0.001$ & Protein degradation \\
\hline Macromolecule catabolic process\| & 111 & 0.40 & 1.92 & 0.034 & $<0.001$ & Protein degradation \\
\hline Protein modification by small protein conjugation\| & 35 & 0.51 & 1.93 & 0.036 & $<0.001$ & Protein degradation \\
\hline Protein ubiquitination\| & 33 & 0.54 & $2 \cdot 01$ & 0.020 & $<0.001$ & Protein degradation \\
\hline Cellular macromolecule catabolic process $\|$ & 83 & 0.46 & 2.05 & 0.013 & $<0.001$ & Protein degradation \\
\hline Ubiquitin cycle\| & 39 & 0.55 & $2 \cdot 18$ & 0.003 & $<0.001$ & Protein degradation \\
\hline Protein catabolic process $\|$ & 56 & 0.59 & 2.43 & 0.000 & $<0.001$ & Protein degradation \\
\hline Cellular protein catabolic process $\|$ & 49 & 0.62 & 2.58 & 0.000 & $<0.001$ & Protein degradation \\
\hline
\end{tabular}

Aqueous green KFE

C57BL/6J

Propanoate metabolism

T-cell receptor signalling pathway

Regulation of immune system process $\|$

Adaptive immune response\|

T-cell activation\|

Adaptive immune response

Haematopoietic cell lineage

Tob1pathwayt†

Antigen processing and presentation

Nktpathway††

Immune system process ||

II12pathwayt†

NO2il12pathwayt†

Cytokine-cytokine receptor interaction

Th1th2pathwayt†

Natural killer cell-mediated cytotoxicity 9

Immune response\|

Hypertrophy model ${ }^{\star *}$

$1110^{-1-}$

P53pathwayt†

DNA replication reactome ${ }^{\star \star}$

Oxidative phosphorylation 9

Pyrimidine metabolism

Proteasome

Proteasomepathway††

Ribosome

Ribosomal proteins ${ }^{* *}$

RNA polymeraseq

Detection of stimulus involved in sensory perception\|

Ethyl acetate gold KFE

C57BL/6J

Cytoskeleton-dependent intracellular transport||

Cholesterol biosynthesis ${ }^{* *}$

$1110^{-1-}$

Cytokine-cytokine receptor interaction 9

Inflampathwayt†

Cytokinepathway $\dagger$

Dcpathway††

Eicosanoid synthesis**

Arachidonic acid metabolism 1

Prostaglandin and leukotriene metabolism

Pyrimidine metabolism

Hypertrophy model ${ }^{* *}$

Ribosomeq

Ribosomal proteins **

RNA polymerase

RNA transcription reactome ${ }^{* *}$

57

22

37

23

67

17

31

28

277

22

16

206

15

91

195

18

16

40

101

82

21

81

21

15

$-0.59$

0.43

0.43
0.48

0.60

0.54

0.61

0.49

0.67

0.58

0.62

0.42

0.66

0.74

0.45

0.78

0.53

0.49

0.61

-0.59
-0.50

$-0.56$

$-0.41$

$-0.63$

$-0.57$

$-0.60$

$-0.54$

$-0.64$

0.76

$-2 \cdot 12$

1.79

1.86

1.88

1.89

1.92

1.96

1.96

1.97

2.05

2.06

2.07

$2 \cdot 13$

$2 \cdot 16$

$2 \cdot 19$

2.25

2.33

$1 \cdot 81$

$-1.77$

$-1.88$

$-2.49$

$-1.78$

$-2.00$

$-1.85$

$-2.51$

$-2.33$

$-2.02$

$2 \cdot 12$

0.013

0.047

0.046

0.043

0.048

0.041

0.007

0.008

0.007

0.003

0.010

0.003

0.001

0.000

0.000

0.000

0.000

0.042

0.041

0.023

0.000

0.043

0.007

0.023

0.000

0.000

0.005

0.007

0.014

0.021

0.022

0.022

0.034

0.032

0.021

0.030

0.048

0.000

0.020

0.001

0.014

0.018

0.029
$<0.001$

$<0.001$

$<0.001$

0.005

0.002

$<0.001$

$<0.001$

$<0.001$

$<0.001$

$<0.001$

$<0.001$

$<0.001$

$<0.001$

$<0.001$

$<0.001$

$<0.001$

$<0.001$

0.003

0.007

$<0.001$

$<0.001$

$<0.001$

0.002

$<0.001$

$<0.001$

$<0.001$

$<0.001$

$<0.001$

$<0.001$

$<0.001$

$<0.001$

0.001

0.010

$<0.001$

0.002

0.001

0.008

$<0.001$

0.002

$<0.001$

$<0.001$

0.002

0.001
Carbohydrate metabolism Immune/inflammation Immune/inflammation Immune/inflammation Immune/inflammation Immune/inflammation Immune/inflammation Immune/inflammation Immune/inflammation Immune/inflammation Immune/inflammation Immune/inflammation Immune/inflammation Immune/inflammation Immune/inflammation Immune/inflammation Immune/inflammation

Tissue remodelling

Cell-cycle arrest

DNA replication

Energy metabolism

Nucleoside metabolism

Protein degradation

Protein degradation

Transcription/translation

Transcription/translation

Transcription/translation

Sensory perception

Cytoskeleton

Lipid metabolism

Immune/inflammation Immune/inflammation Immune/inflammation Immune/inflammation

Lipid metabolism

Lipid metabolism

Lipid metabolism

Nucleoside metabolism

Tissue remodelling

Transcription/translation

Transcription/translation

Transcription/translation

Transcription/translation 
Table 4. Continued

\begin{tabular}{|c|c|c|c|c|c|c|}
\hline MSigDB gene set name† & Size & ES & NES $\ddagger$ & FDR $q$-value§ & FE $P \S$ & Function \\
\hline Selenoamino acid metabolism & 19 & 0.66 & 2.09 & 0.005 & $<0.001$ & Amino acid metabolism \\
\hline Valine, leucine and isoleucine degradation & 41 & 0.53 & 2.09 & 0.005 & $<0.001$ & Amino acid metabolism \\
\hline Gluconeogenesis** & 44 & 0.42 & 1.66 & 0.049 & $<0.001$ & Carbohydrate metabolism \\
\hline Glycolysis** & 44 & 0.42 & 1.67 & 0.047 & 0.006 & Carbohydrate metabolism \\
\hline Propanoate metabolism & 29 & 0.48 & $1 \cdot 70$ & 0.041 & 0.010 & Carbohydrate metabolism \\
\hline Insulin signalling pathway & 120 & 0.37 & $1 \cdot 78$ & 0.032 & $<0.001$ & Carbohydrate metabolism \\
\hline Glycolysis and gluconeogenesis & 51 & 0.45 & $1 \cdot 86$ & 0.020 & $<0.001$ & Carbohydrate metabolism \\
\hline Krebs/TCA cycle c** $^{\star *}$ & 27 & 0.60 & 2.08 & 0.006 & $<0.001$ & Carbohydrate metabolism \\
\hline Citrate cycle & 23 & 0.75 & 2.51 & 0.000 & $<0.001$ & Carbohydrate metabolism \\
\hline Focal adhesion & 180 & 0.40 & 2.01 & 0.009 & $<0.001$ & Cell adhesion \\
\hline Integrin-mediated cell adhesion KEGG & 88 & 0.45 & 2.03 & 0.008 & $<0.001$ & Cell adhesion \\
\hline Integrinpathway†† & 33 & 0.58 & $2 \cdot 11$ & 0.005 & $<0.001$ & Cell adhesion \\
\hline Ecmpathway†† & 19 & 0.69 & $2 \cdot 17$ & 0.003 & $<0.001$ & Cell adhesion \\
\hline Striated muscle contraction ${ }^{\star *}$ & 32 & 0.46 & $1 \cdot 64$ & 0.050 & 0.003 & Cytoskeleton \\
\hline Regulation of actin cytoskeleton & 182 & 0.33 & $1 \cdot 66$ & 0.050 & $<0.001$ & Cytoskeleton \\
\hline Mitochondriapathway†† & 20 & 0.53 & $1 \cdot 70$ & 0.041 & 0.005 & Energy metabolism \\
\hline Oxidative phosphorylation & 101 & 0.41 & $1 \cdot 89$ & 0.017 & $<0.001$ & Energy metabolism \\
\hline Cellular respiration\| & 19 & 0.64 & 2.07 & 0.017 & $<0.001$ & Energy metabolism \\
\hline Fatty acid metabolic process\| & 43 & 0.49 & 1.95 & 0.037 & $<0.001$ & Lipid metabolism \\
\hline Fatty acid oxidation\| & 15 & 0.68 & 1.97 & 0.043 & 0.005 & Lipid metabolism \\
\hline Fatty acid metabolism & 39 & 0.53 & 1.99 & 0.011 & $<0.001$ & Lipid metabolism \\
\hline Lipid transport\| & 24 & 0.66 & $2 \cdot 30$ & 0.002 & $<0.001$ & Lipid metabolism \\
\hline Energy derivation by oxidation of organic compounds\| & 35 & 0.64 & $2 \cdot 42$ & 0.000 & $<0.001$ & Lipid metabolism \\
\hline Flagellar assembly & 20 & 0.52 & $1 \cdot 68$ & 0.046 & 0.008 & Motility \\
\hline Pyk2pathway†† & 26 & 0.56 & 1.95 & 0.014 & 0.000 & Signalling \\
\hline Raspathway†† & 20 & 0.61 & 1.98 & 0.012 & 0.003 & Signalling \\
\hline Calcium regulation in cardiac cells ${ }^{* *}$ & 136 & 0.37 & $1 \cdot 80$ & 0.030 & $<0.001$ & Signalling, calcium \\
\hline Chrebppathway†† & 18 & 0.57 & $1 \cdot 80$ & 0.031 & 0.008 & Signalling, carbohydrate \\
\hline Egfpathway†† & 24 & 0.59 & $1 \cdot 73$ & 0.038 & 0.006 & Signalling, proliferation \\
\hline Cxcr4pathway†† & 21 & 0.47 & 1.90 & 0.016 & $<0.001$ & Signalling, chemokine \\
\hline Wnt signalling $\ddagger \ddagger$ & 55 & 0.52 & 1.93 & 0.012 & $<0.001$ & Signalling, development \\
\hline Pgc1apathway†† & 23 & 0.48 & 1.73 & 0.037 & 0.003 & Signalling, energy metabolism \\
\hline G-protein signalling ${ }^{\star \star}$ & 84 & 0.54 & $2 \cdot 14$ & 0.005 & $<0.001$ & Signalling, G-protein \\
\hline Gpcrpathway†† & 30 & 0.46 & 1.94 & 0.013 & $<0.001$ & Signalling, GPC receptor \\
\hline P38mapkpathway†† & 37 & 0.49 & $1 \cdot 72$ & 0.038 & 0.006 & Signalling, inflammation \\
\hline Tcrpathway†† & 42 & 0.52 & $1 \cdot 85$ & 0.021 & $<0.001$ & Signalling, inflammation \\
\hline Fcer1pathway†† & 36 & 0.53 & 1.94 & 0.013 & $<0.001$ & Signalling, inflammation \\
\hline Bcrpathway†† & 32 & 0.62 & 1.95 & 0.015 & $<0.001$ & Signalling, inflammation \\
\hline Vippathway†† & 25 & 0.44 & $2 \cdot 12$ & 0.005 & $<0.001$ & Signalling, inflammation \\
\hline Ppar signalling pathway & 61 & 0.53 & $1 \cdot 82$ & 0.025 & $<0.001$ & Signalling, lipid \\
\hline Ptdinspathway†† & 21 & 0.47 & 1.75 & 0.036 & 0.005 & Signalling, lipid kinase \\
\hline Phosphatidylinositol signalling system & 65 & 0.39 & 2.05 & 0.007 & $<0.001$ & Signalling, lipid kinase \\
\hline Mapkpathway†† & 81 & 0.54 & $1 \cdot 76$ & 0.035 & $<0.001$ & Signalling, MAP kinase \\
\hline Mcalpainpathway†† & 20 & 0.57 & $1 \cdot 74$ & 0.037 & 0.007 & Signalling, motility \\
\hline Ngfpathway†† & 16 & 0.40 & $1 \cdot 76$ & 0.035 & 0.010 & Signalling, nerve growth \\
\hline Axon guidance & 118 & 0.51 & 1.94 & 0.012 & $<0.001$ & Signalling, nerve growth \\
\hline Biopeptidespathway†† & 36 & 0.55 & 1.92 & 0.013 & $<0.001$ & Signalling, peptide \\
\hline Tpopathway†† & 21 & 0.54 & $1 \cdot 76$ & 0.035 & $<0.001$ & Signalling, platelets \\
\hline Sppapathway†† & 19 & 0.60 & $1 \cdot 74$ & 0.037 & 0.010 & Signalling, platelets \\
\hline Par1pathway†† & 18 & 0.56 & $1 \cdot 87$ & 0.018 & 0.003 & Signalling, platelets \\
\hline Pdgfpathway†† & 24 & 0.52 & $1 \cdot 88$ & 0.019 & $<0.001$ & Signalling, platelets \\
\hline \multicolumn{7}{|l|}{ Ethyl acetate green KFE } \\
\hline \multicolumn{7}{|l|}{ C57BL/6J } \\
\hline Striated muscle contraction** & 32 & -1.99 & -1.99 & 0.027 & $<0.001$ & Cytoskeleton \\
\hline Type I diabetes mellitus $\rrbracket$ & 22 & 0.57 & $1 \cdot 88$ & 0.028 & $<0.001$ & Carbohydrate metabolism \\
\hline Dcpathway†† & 21 & 0.55 & $1 \cdot 77$ & 0.048 & 0.005 & Immune/inflammation \\
\hline T-cell receptor signalling pathway 9 & 88 & 0.40 & $1 \cdot 82$ & 0.038 & $<0.001$ & Immune/inflammation \\
\hline Cytokinepathway†† & 20 & 0.58 & $1 \cdot 85$ & 0.033 & $<0.001$ & Immune/inflammation \\
\hline Haematopoietic cell lineage & 67 & 0.43 & $1 \cdot 86$ & 0.031 & $<0.001$ & Immune/inflammation \\
\hline Calcineurin/NFAT signallingł & 89 & 0.44 & 1.96 & 0.019 & $<0.001$ & Immune/inflammation \\
\hline Antigen processing and presentation & 31 & 0.56 & 1.99 & 0.017 & $<0.001$ & Immune/inflammation \\
\hline Ctla4pathway†† & 17 & 0.67 & $2 \cdot 00$ & 0.018 & 0.003 & Immune/inflammation \\
\hline NO2il12pathway†† & 16 & 0.71 & $2 \cdot 11$ & 0.006 & $<0.001$ & Immune/inflammation \\
\hline II12pathway†† & 22 & 0.65 & $2 \cdot 13$ & 0.005 & $<0.001$ & Immune/inflammation \\
\hline Natural killer cell-mediated cytotoxicity & 91 & 0.48 & $2 \cdot 13$ & 0.009 & $<0.001$ & Immune/inflammation \\
\hline Biosynthesis of steroids & 23 & 0.55 & $1 \cdot 82$ & 0.036 & 0.008 & Lipid metabolism \\
\hline Cholesterol biosynthesis ${ }^{\star *}$ & 15 & 0.65 & 1.91 & 0.022 & $<0.001$ & Lipid metabolism \\
\hline Proteasomepathway†† & 21 & 0.54 & $1 \cdot 76$ & 0.050 & 0.005 & Protein degradation \\
\hline Proteasomeף & 20 & $0 \cdot 70$ & $1 \cdot 83$ & 0.036 & 0.007 & Protein degradation \\
\hline
\end{tabular}


Table 4. Continued

\begin{tabular}{|c|c|c|c|c|c|c|}
\hline MSigDB gene set name $†$ & Size & ES & NES $\ddagger$ & FDR $q$-value§ & FE $P \S$ & Function \\
\hline Basal transcription factors & 26 & 0.52 & 1.75 & 0.050 & 0.005 & Transcription/translation \\
\hline $\begin{array}{l}\text { RNA transcription reactome }{ }^{* *} \\
I / 10^{-/-}\end{array}$ & 36 & 0.51 & 1.94 & 0.019 & $<0.001$ & Transcription/translation \\
\hline Valine, leucine and isoleucine degradation & 41 & -0.61 & $-2 \cdot 26$ & 0.000 & $<0.001$ & Amino acid metabolism \\
\hline Propanoate metabolism & 29 & -0.66 & $-2 \cdot 27$ & 0.000 & $<0.001$ & Carbohydrate metabolism \\
\hline Citrate cycleq & 23 & -0.63 & -2.07 & 0.002 & $<0.001$ & Carbohydrate metabolism \\
\hline Krebs/TCA cycle** & 27 & -0.57 & -1.92 & 0.012 & 0.002 & Carbohydrate metabolism \\
\hline Pyruvate metabolism & 34 & -0.52 & -1.86 & 0.019 & 0.002 & Carbohydrate metabolism \\
\hline Coenzyme metabolic process $\|$ & 25 & -0.61 & -2.05 & 0.006 & 0.002 & Coenzyme metabolism \\
\hline Regulation of muscle contraction\| & 17 & -0.63 & $-1 \cdot 88$ & 0.025 & 0.002 & Cytoskeleton \\
\hline Response to oxidative stress $\|$ & 40 & -0.57 & $-2 \cdot 16$ & 0.001 & $<0.001$ & Detoxification \\
\hline Digestion\| & 36 & -0.54 & -1.97 & 0.013 & $<0.001$ & Digestion \\
\hline Oxidative phosphorylation & 101 & -0.62 & $-2 \cdot 80$ & 0.000 & $<0.001$ & Energy metabolism \\
\hline Cellular respiration\| & 19 & -0.69 & $-2 \cdot 20$ & 0.002 & $<0.001$ & Energy metabolism \\
\hline Aerobic respiration\| & 15 & -0.67 & -1.95 & 0.014 & 0.004 & Energy metabolism \\
\hline Carbon fixation & 19 & -0.61 & $-1 \cdot 88$ & 0.017 & 0.002 & Energy metabolism \\
\hline Energy derivation by oxidation of organic compounds\| & 35 & -0.55 & $-2 \cdot 00$ & 0.010 & $<0.001$ & Lipid metabolism \\
\hline Fatty acid metabolism & 39 & -0.49 & $-1 \cdot 81$ & 0.027 & 0.005 & Lipid metabolism \\
\hline Glutathione metabolism & 34 & -0.63 & $-2 \cdot 26$ & 0.000 & $<0.001$ & Xenobiotic metabolism \\
\hline Metabolism of xenobiotics by cytochrome P450 & 35 & -0.60 & $-2 \cdot 17$ & 0.000 & $<0.001$ & Xenobiotic metabolism \\
\hline Cell-cycle phase\|l & 139 & 0.40 & 1.81 & 0.047 & $<0.001$ & Cell proliferation \\
\hline M phase $\|$ & 93 & 0.43 & 1.82 & 0.047 & $<0.001$ & Cell proliferation \\
\hline Mitosis\| & 66 & 0.47 & 1.86 & 0.034 & $<0.001$ & Cell proliferation \\
\hline Chromatin assembly or disassembly\|l & 24 & 0.58 & 1.87 & 0.037 & $<0.001$ & Cell proliferation \\
\hline Cytokinesis\| & 17 & 0.66 & 1.89 & 0.034 & $<0.001$ & Cell proliferation \\
\hline Mitotic cell cycle\| & 124 & 0.43 & 1.91 & 0.044 & $<0.001$ & Cell proliferation \\
\hline M phase of mitotic cell cycle\|l & 69 & 0.48 & 1.94 & 0.035 & $<0.001$ & Cell proliferation \\
\hline Cell division\| & 19 & 0.64 & 1.95 & 0.048 & $<0.001$ & Cell proliferation \\
\hline Microtubule cytoskeleton organisation and biogenesis $\|$ & 26 & 0.56 & 1.84 & 0.042 & $<0.001$ & Cytoskeleton \\
\hline Cytokine-cytokine receptor interaction & 206 & 0.42 & $2 \cdot 01$ & 0.020 & $<0.001$ & Immune/inflammation \\
\hline SA MMP cytokine connection§§ & 15 & 0.77 & $2 \cdot 17$ & 0.003 & $<0.001$ & Immune/inflammation \\
\hline RNA processing\| & 130 & 0.42 & 1.88 & 0.033 & $<0.001$ & Transcription/translation \\
\hline RNA catabolic process $\|$ & 16 & 0.66 & $1 \cdot 89$ & 0.041 & 0.002 & Transcription/translation \\
\hline
\end{tabular}

ES, enrichment score assigned to reflect the degree to which a gene set was over-represented in the top or bottom of the ranked list; NES, enrichment score normalised for differences in gene set size; FDR $q$-value, false discovery rate; FE $P$ value, Fisher's exact test significance level; MAP, mitogen-activated protein.

* GSEA was applied as described ${ }^{(40)}$ to identify up- and down-regulated processes after feeding a diet supplemented with KFE compared with a control diet.

† All gene sets were downloaded from the MSigDB database version 2.5 on 29 November 2009 (http://www.broadinstitute.org/gsea/msigdb/) ${ }^{(41)}$.

$\ddagger N E S>0$ is associated with the control diet; NES $<0$ is associated with the KFE-supplemented diet.

$\S$ Gene set enrichment was considered significant when FDR $q$ value $\leq 0.05$ and FE $P$ value $\leq 0.01$.

\| Original gene set source as listed by MSigDB: Gene Ontology.

ๆ Original gene set source as listed by MSigDB: KEGG (Kyoto Encyclopedia of Genes and Genomes; http://www.genome.jp/kegg/).

** Original gene set source as listed by MSigDB: Wiki Pathways.

†† Original gene set source as listed by MSigDB: BioCarta; Gene arrays.

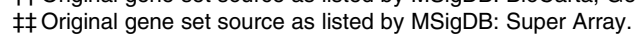

$\S \S$ Original gene set source as listed by MSigDB: Sigma-Aldrich.

metabolism were decreased in the colon samples from $\mathrm{Il1 \textrm {O } ^ { - / - }}$ mice fed the same diets. However, the protein degradation pathway appeared to be differently regulated between the gold and green aqueous KFE, with increased expression in gene sets related to ubiquitination and degradation in colon samples from $\mathrm{IllO}^{-1-}$ mice fed the gold aqueous KFE diet, but decreased expression levels across gene sets related to the proteasome in colon samples from $\mathrm{Il1O}^{-/-}$mice fed the green aqueous KFE diet.

Colon samples from $\mathrm{Il1O}^{-/-}$mice fed the gold ethyl acetate extract showed increased expression levels across gene sets related to inflammation and eicosanoid synthesis when compared with those from mice fed the control diet. This was accompanied by decreased expression levels across gene sets related to carbohydrate, amino acid and lipid metabolism, as well as a range of signalling pathways such as G-protein-coupled and G-protein-coupled receptor signalling, cell adhesion, growth factor, mitogen-activated protein kinase (MAPK) and lipid kinase signalling.

Expression levels across gene sets related to immune and inflammatory signalling were decreased in colon samples from both $\mathrm{C} 57 \mathrm{BL} / 6 \mathrm{~J}$ and $I l 10^{-1-}$ mice fed the green ethyl acetate extract when compared with samples from mice fed the control diet. The pathways associated with gene sets enriched in C57BL/6J colon samples included T-cell and dendritic cell signalling, antigen processing, and the IL12 pathway, whereas those within the $1110^{-/-}$colon samples were associated with cytokine signalling.

All genes chosen for qRT-PCR validation of relative expression between the treatment groups showed similar FC in both microarray and qRT-PCR analyses (Fig. 1). Expression levels of four genes involved in the inflammatory processes present within the colon (Mmp10, Mmp13, Reg3b and $5100 a 8$ ) were increased in colon samples from $1110^{-1-}$ mice compared with those of C57BL/6J mice for both 
NS British Journal of Nutrition

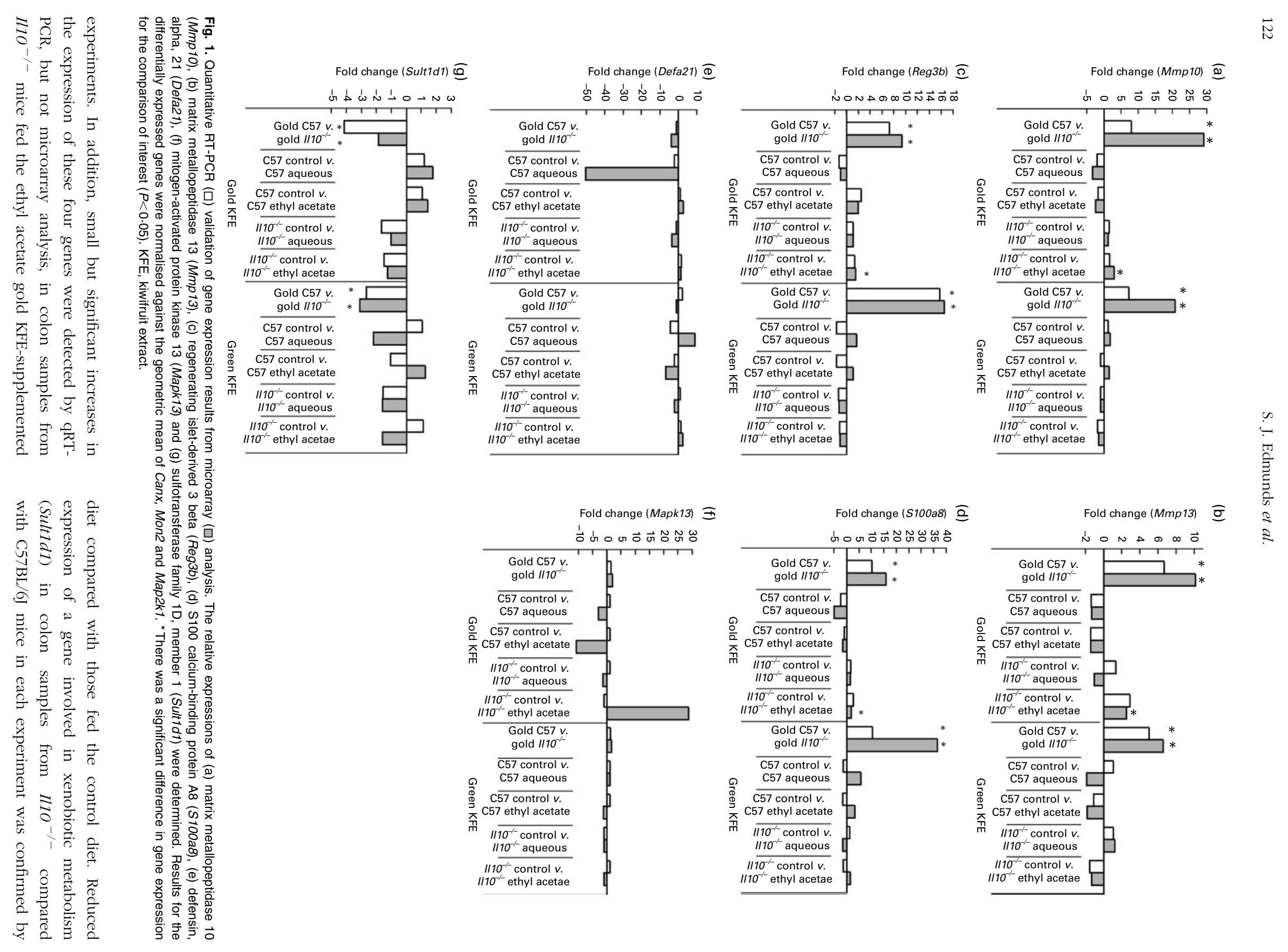


qRT-PCR. Only two genes (Mapk13 and Defa21) were chosen that were not differentially expressed between the treatment groups when measured by microarray analysis, and the absence of differential expression was confirmed by qRT-PCR.

\section{Changes in colonic protein abundances}

Images from thirteen of the sixteen gels were captured successfully and relative expression levels were analysed using DIGE, where the two protein samples were directly compared within each gel. The data from two gels comparing $110^{-/-}$ mice fed the aqueous gold KFE diet with those fed the gold control diet and from one gel comparing C57BL/6J mice fed the ethyl acetate green KFE diet with those fed the green control diet could not be used because of technical error. Therefore, these comparisons were conducted between the appropriate samples in different gels. Image warping ensured that protein spots were compared correctly between gels and false positive results were unlikely. However, as this analysis is less sensitive, there was an increased chance of a false negative result where a differentially expressed protein would not appear to have a significant FC.

A total of sixty-one protein spots were identified as differentially expressed as a result of the inclusion of KFE in the diet (Table 5), with gel locations indicated for differentially expressed proteins in the $\mathrm{C} 57 \mathrm{BL} / 6 \mathrm{~J}$ and $1110^{-/-}$samples (Fig. 2). Of these, forty-eight spots were successfully identified by MS as corresponding to a single protein and two spots were identified by MS as having two possible protein matches (see Table S1 of the supplementary material, available online at http://www.journals.cambridge.org/bjn). For the latter two spots, the two possible proteins had similar functions; therefore, both identifications were retained. A further eleven spots were identified by comparison with a reference gel image compiled from previous experiments by our research group, which used $1 l 1 \mathrm{O}^{-/-}$and $\mathrm{C} 57 \mathrm{BL} / 6 \mathrm{~J}$ mice fed an AIN76A control diet (see Table S2 of the supplementary material, available online at http://www.journals.cambridge.org/bjn). In many cases, the same protein ID was matched to more than one spot, indicating that different isoforms of that protein were present, probably because of post-translational modification. The FC for all isoforms of each protein were in the same direction; however, not all isoforms were differentially expressed in the same comparisons.

Abundances of negative acute-phase proteins were decreased in the colon samples from $\mathrm{C} 57 \mathrm{BL} / 6 \mathrm{~J}$ and $1110^{-1-}$ mice fed the gold aqueous extract when compared with those fed the control diet (Table 5). This included transferrin, a protein known to be increased within the colon tissue during inflammation. In addition, decreased abundances of the molecular chaperone proteins, PDIA3, PPIA and CALR, and of proteins involved in carbohydrate metabolism were detected in the colon samples from $\mathrm{Il1O}^{-/-}$mice fed this extract. Decreased abundances of molecular chaperon proteins (HSPA8 and HSPD1), proteins involved in carbohydrate and energy metabolism, and cytoskeleton components were also detected in colon samples from $\mathrm{Il1O}^{-/-}$mice fed the green aqueous and green ethyl acetate extracts when compared with samples from mice fed the appropriate control diet.

\section{Discussion}

\section{Effects of kiwifruit extracts on animal health and inflammation}

The histological investigation of colon tissues collected from $\mathrm{C} 57 \mathrm{BL} / 6 \mathrm{~J}$ and $I l 10^{-/-}$mice after the dietary intervention studies indicates that $1 l 10^{-/-}$mice develop significant colitis 6 weeks after inoculation with a mixture of normal intestinal bacteria. This is supported by reduced weight gain, overall loss of condition and increased plasma concentrations of the acute-phase biomarker IL-6 in $1 l 10^{-/}$mice. These findings are similar to previous reports using this inoculated model $^{(25-27)}$ and are also similar to the pathophysiology present in inflamed intestinal tissue in Crohn's disease patients $^{(1)}$

As the addition of KFE to the diet of $\mathrm{C} 57 \mathrm{BL} / 6 \mathrm{~J}$ or $1110^{-1-}$ mice does not alter inflammatory parameters, KFE do not appear to have an anti-inflammatory effect in this animal model. Therefore, the immune-suppressing activity previously demonstrated in vitro does not translate into this in vivo model of IBD. This may be due to the effects of the intestinal environment on the KFE. For example, potentially anti-inflammatory polyphenols present within the KFE often have low bioavailability in vivo ${ }^{(49)}$. However, Halliwell et al. ${ }^{(50)}$ have shown that, while ingestion of polyphenols typically leads to low maximal plasma concentrations $(<1 \mu \mathrm{mol} / \mathrm{ml})$, a much higher concentration of polyphenols is present in the intestinal lumen where they can interact directly with the intestinal mucosa. This interaction is expected to negate any absence of systemic activity caused by low absorption across the intestinal mucosa. In addition, metabolites from the KFE were detected within the urine of all mice fed KFE-supplemented diets within these experiments ${ }^{(51)}$, indicating that at least some KFE compounds are digested, absorbed and metabolised by mice. Therefore, it is unlikely that the absence of anti-inflammatory activity is due to low bioavailability alone. As the physiological complexity present in vivo is not present in single-cell in vitro assays, the KFE compounds present in these animal models may be substantially different from those previously tested.

\section{Molecular effects of kiwifruit extracts}

Detailed investigations of the effects of the KFE intervention on gene or protein expression levels found the effects on both inflammatory signalling and other metabolic processes within colonic tissue collected from both $\mathrm{C} 57 \mathrm{BL} / 6 \mathrm{~J}$ and $I l 1 \mathrm{O}^{-1-}$ mice; however, these effects were subtle. While no significant changes in individual gene expression were identified by linear models for microarray data (LIMMA) analysis, GSEA analysis identified between two and sixty-four significantly enriched gene sets in each comparison. The aim of GSEA is to identify groups of functionally related genes with 
Table 5. Proteins more or less abundant in the colon of mice fed the kiwifruit extract (KFE)-supplemented diets compared with mice fed a control diet

\begin{tabular}{|c|c|c|c|c|}
\hline \multirow[b]{2}{*}{ Spot no.† } & \multirow[b]{2}{*}{ Symbolł } & \multicolumn{2}{|c|}{$\mathrm{FC}^{*}$} & \multirow[b]{2}{*}{ Function } \\
\hline & & C57 & $1110^{-1-}$ & \\
\hline \multicolumn{5}{|c|}{ Aqueous gold $\mathrm{KFE}$} \\
\hline 15 & REG3B & & $-4 \cdot 1$ & Acute-phase protein, LPS regulated \\
\hline 2 & TRF & $-2 \cdot 3$ & $-15 \cdot 9$ & Negative acute-phase protein, Fe transport \\
\hline 8 & TRF & $-2 \cdot 2$ & $-16 \cdot 0$ & Negative acute-phase protein, Fe transport \\
\hline 11 & TRF & $-2 \cdot 1$ & & Negative acute-phase protein, Fe transport \\
\hline 12 & TRF & $-2 \cdot 3$ & & Negative acute-phase protein, Fe transport \\
\hline 31 & TRF & $-2 \cdot 2$ & $-14 \cdot 6$ & Negative acute-phase protein, Fe transport \\
\hline 30 & ALB & & $-2 \cdot 1$ & Negative acute-phase protein, serum protein \\
\hline 54 & ALB & & -3.5 & Negative acute-phase protein, serum protein \\
\hline 62 & ALB & -2.4 & & Negative acute-phase protein, serum protein \\
\hline 53 & PPIA & & -3.0 & Protein folding, LPS signalling \\
\hline 32 & PDIA3 & $-2 \cdot 0$ & $-7 \cdot 8$ & Protein folding, oxidative stress \\
\hline 60 & PDIA3 & $-2 \cdot 0$ & $-4 \cdot 2$ & Protein folding, oxidative stress \\
\hline 50 & CFL1 & & $-5 \cdot 4$ & Cytoskeleton, actin regulation \\
\hline 3 & MYL6 & $-2 \cdot 2$ & & Cytoskeleton, muscle fibre \\
\hline 1 & TPM2 & $-2 \cdot 4$ & & Cytoskeleton, actin filament \\
\hline 29 & ARHGDIA & & $-5 \cdot 7$ & RhoGTPase inhibition \\
\hline 6 & CAR1 & & $-2 \cdot 6$ & Ion transport, $\mathrm{pH}$ homeostasis \\
\hline 13 & CAR1 & & $-2 \cdot 6$ & Ion transport, $\mathrm{pH}$ homeostasis \\
\hline 27 & CAR1 & & $-2 \cdot 8$ & Ion transport, $\mathrm{pH}$ homeostasis \\
\hline 28 & CAR1 & & $-2 \cdot 8$ & Ion transport, $\mathrm{pH}$ homeostasis \\
\hline 61 & TALDO1 & $-2 \cdot 1$ & & Pentose phosphate pathway \\
\hline 52 & GAPDH & & $-15 \cdot 5$ & Glycolysis \\
\hline 56 & MDH1 & & -4.0 & Citric acid cycle \\
\hline 23 & EPHX1 & $-2 \cdot 6$ & $-18 \cdot 1$ & Xenobiotic metabolism \\
\hline 17 & HNRNPAB & $-2 \cdot 1$ & $-5 \cdot 8$ & Transcription regulation \\
\hline 55 & HNRNPA2B1 & & $-6 \cdot 6$ & Transcription regulation \\
\hline 73 & EIF5A & & $-8 \cdot 7$ & Translation, apoptosis regulation \\
\hline 104 & EIF5A & & $-9 \cdot 7$ & Translation, apoptosis regulation \\
\hline \multicolumn{5}{|c|}{ Aqueous green $\mathrm{KFE}$} \\
\hline 107 & CALR & & & Protein folding, apoptosis, antigen presentation \\
\hline 44 & HSPA8 & & $-2 \cdot 2$ & Protein folding, LPS signalling \\
\hline 46 & HSPA8 & & $-2 \cdot 4$ & Protein folding, LPS signalling \\
\hline 49 & HSPD1 & & $-2 \cdot 3$ & Protein folding, T-cell activation, TLR signalling \\
\hline 106 & HSPD1 & & $-2 \cdot 2$ & Protein folding, T-cell activation, TLR signalling \\
\hline 3 & MYL6 & $-2 \cdot 7$ & & Cytoskeleton, muscle fibre \\
\hline 1 & TPM2 & $-2 \cdot 3$ & & Cytoskeleton, actin filament \\
\hline 7 & ACTA2 & & $-2 \cdot 2$ & Cytoskeleton, actin filament \\
\hline 91 & CAP1 & & $-2 \cdot 7$ & Cytoskeleton, actin filament \\
\hline 93 & DES & & $-2 \cdot 1$ & Cytoskeleton, intermediate filament \\
\hline 94 & DES & & $-2 \cdot 3$ & Cytoskeleton, intermediate filament \\
\hline 42 & TUBA1A & $-2 \cdot 0$ & & Cytoskeleton, microtubule/intermediate filament \\
\hline 10 & ATP5B & & -2.5 & ATP synthesis \\
\hline 21 & ATP5A1 & & $-2 \cdot 2$ & ATP synthesis \\
\hline 43 & ATP5A1 & & $-2 \cdot 1$ & ATP synthesis \\
\hline 92 & TKT & & -3.9 & Pentose phosphate pathway \\
\hline 97 & IDH1 & & $-2 \cdot 2$ & Citric acid cycle \\
\hline 95 & LDHA & & $-2 \cdot 0$ & Glycolysis, pyruvate metabolism \\
\hline 22 & PKM2 & & $-4 \cdot 0$ & Pyruvate metabolism \\
\hline 96 & PKM2 & & $-4 \cdot 6$ & Pyruvate metabolism \\
\hline 41 & ALDH1B1 & & -3.0 & Alcohol metabolism, lipid metabolism \\
\hline 33 & SERPINB1A & & $-2 \cdot 2$ & Protein catabolism, peptidase inhibitor \\
\hline 76 & SELENBP1 & & -4.5 & Selenium binding, protein transport \\
\hline \multicolumn{5}{|c|}{ Ethyl gold acetate KFE } \\
\hline 3 & MYL6 & $-4 \cdot 1$ & $-4 \cdot 7$ & Cytoskeleton, muscle fibre \\
\hline 1 & TPM2 & $-2 \cdot 2$ & $-2 \cdot 6$ & Cytoskeleton, actin filament \\
\hline 42 & TUBA1A & $-2 \cdot 0$ & & Cytoskeleton, microtubule/intermediate filament \\
\hline 23 & EPHX1 & -1.9 & & Xenobiotic metabolism \\
\hline \multicolumn{5}{|c|}{ Ethyl acetate green KFE } \\
\hline 15 & REG3B & & 1.8 & Acute-phase protein, LPS regulated \\
\hline 84 & HSPA5 & & $-2 \cdot 2$ & Protein folding \\
\hline 44 & HSPA8 & & $-2 \cdot 6$ & Protein folding, LPS signalling \\
\hline 46 & HSPA8 & & $-2 \cdot 8$ & Protein folding, LPS signalling \\
\hline 49 & HSPD1 & -1.8 & $-3 \cdot 7$ & Protein folding, T-cell activation, TLR signalling \\
\hline 106 & HSPD1 & & -2.9 & Protein folding, T-cell activation, TLR signalling \\
\hline 3 & MYL6 & $-2 \cdot 2$ & & Cytoskeleton, smooth muscle fibre \\
\hline 7 & ACTA2 & & $-2 \cdot 2$ & Cytoskeleton, actin filament \\
\hline
\end{tabular}


Table 5. Continued

\begin{tabular}{lllll}
\hline & & \multicolumn{2}{c}{ FC $^{*}$} & \\
\cline { 2 - 3 } Spot no.† & Symbol & C57 & $110^{-1-}$ & Function \\
\hline \multirow{2}{*}{82} & ANXA2 & & $-2 \cdot 0$ & Cytoskeleton, macrophage activation \\
51 & KRT8 & & $-2 \cdot 4$ & Cytoskeleton, intermediate filament \\
68 & KRT8 & & $-3 \cdot 1$ & Cytoskeleton, intermediate filament \\
80 & KRT20 & & $-1 \cdot 8$ & Cytoskeleton, intermediate filament \\
81 & KRT20 & & $-3 \cdot 4$ & Cytoskeleton, intermediate filament \\
42 & TUBA1A & $-2 \cdot 0$ & & Cytoskeleton, microtubule/intermediate filament \\
85 & DPYSL2 & & $-2 \cdot 7$ & Axonal development, T-cell response \\
88 & GDI2 & & $-2 \cdot 4$ & GTPase inhibitor, protein transport \\
26 & CKB & & $-2 \cdot 1$ & Energy metabolism, osteoclast activity \\
10 & ATP5B & $-4 \cdot 2$ & $-5 \cdot 6$ & ATP synthesis \\
21 & ATP5A1 & & $-3 \cdot 2$ & ATP synthesis \\
43 & ATP5A1 & & $-3 \cdot 5$ & ATP synthesis \\
92 & TKT & & $-4 \cdot 0$ & Pentose phosphate pathway \\
67 & MDH1 & & $-1 \cdot 9$ & Citric acid cycle \\
97 & IDH1 & & $-2 \cdot 7$ & Citric acid cycle \\
83 & PGK1 & & $-2 \cdot 0$ & Glycolysis \\
86 & ENO1 & & $-2 \cdot 1$ & Glycolysis \\
22 & PKM2 & & $-6 \cdot 5$ & Pyruvate metabolism \\
96 & PKM2 & & $-3 \cdot 9$ & Pyruvate metabolism \\
41 & ALDH1B1 & & $-3 \cdot 0$ & Alcohol metabolism, lipid metabolism \\
33 & SERPINB1A & $-1 \cdot 7$ & $-2 \cdot 1$ & Protein catabolism, peptidase inhibitor \\
76 & SELENBP1 & & $-2 \cdot 9$ & Se binding, protein transport \\
\hline
\end{tabular}

FC, fold change, LPS, lipopolysaccharide; TLR, Toll-like receptor.

* Differential expression was based on mean FC from two gels, representing six biological replicates. Differential expression was considered significant when one of two biological replicates $|\mathrm{FC}|>2.0$ and the second replicate $|\mathrm{FC}|>1.3$ in the same direction. † Refer to Fig. 2.

‡ Mouse Genome Informatics (http://www.informatics.jax.org/). For a description of the full names of the proteins, see http://www. genecards.org/

statistically significant, coordinated changes in expression even when no individual genes would be identified by individual gene analysis ${ }^{(52)}$. The GSEA-P analysis tool (http://www.broadinstitute.org/gsea/index.jsp) was used by Mootha et $a l .{ }^{(42)}$ to identify a specific set of genes related to oxidative phosphorylation as differentially regulated in muscle tissue from type 2 diabetics, a finding which was then validated in independent studies despite no significant differences in individual expression identified for these genes. Therefore, both significantly enriched gene sets and differentially expressed proteins will be discussed for each comparison.

\section{Aqueous gold kiwifruit extract}

The aqueous gold KFE appears to have an immune-suppressing effect within the colon of $\mathrm{C} 57 \mathrm{BL} / 6 \mathrm{~J}$, but not $1110^{-/-}$, mice, with decreased expression levels across three sets of genes related to immune function and inflammation compared with levels found in mice fed a KFE-free control diet. This is supported by the reduced protein abundance of transferrin, an $\mathrm{Fe}$ transport protein that increases within the colon in response to inflammation, and of three molecular chaperone proteins associated with cellular stress and TLR signalling (PDIA3, PPIA and CALR), within these colon samples (Table 5). However, the absence of colon transcript changes or a reduction in colon HIS in $1 l 10^{-/-}$mice indicates that there is no anti-inflammatory effect within these animals.
While inflammation within the $I l 10^{-/-}$mouse colon is not reduced by supplementation with the aqueous gold KFE, this extract appears to reduce the overall metabolic processes within these tissues compared with similar mice fed the control diet. Both transcriptomic and proteomic data indicate a reduction in carbohydrate and energy metabolism, coupled with decreases in gene set expression related to protein ubiquitination and degradation. The suppression of protein ubiquitination may be related to the reduced abundance of molecular chaperone proteins involved in facilitating protein folding within the endoplasmic reticulum. Under conditions of oxidative stress, such as that reported within the $1110^{-1-}$ mouse colon ${ }^{(53)}$, proteins may become misfolded to form non-functional protein aggregates ${ }^{(54)}$. The abnormal proteins are ubiquitinated and then degraded by the proteasome complex ${ }^{(55)}$. As protein folding is linked to de novo expression ${ }^{(56)}$, reduced protein expression due to decreased metabolic capacity may also reduce the need for molecular chaperones and protein degradation.

\section{Aqueous green kiwifruit extract}

Similar results were found for the aqueous green KFE to aqueous gold KFE, with decreased expression levels across sixteen gene sets related to the adaptive immune response and T-cell activation within colon samples from $\mathrm{C} 57 \mathrm{BL} / 6 \mathrm{~J}$, but not $I l 1 O^{-/-}$, mice fed the KFE-supplemented diet. Other mouse studies have demonstrated that gold and green kiwifruit enhance the adaptive immune response to 
(a)

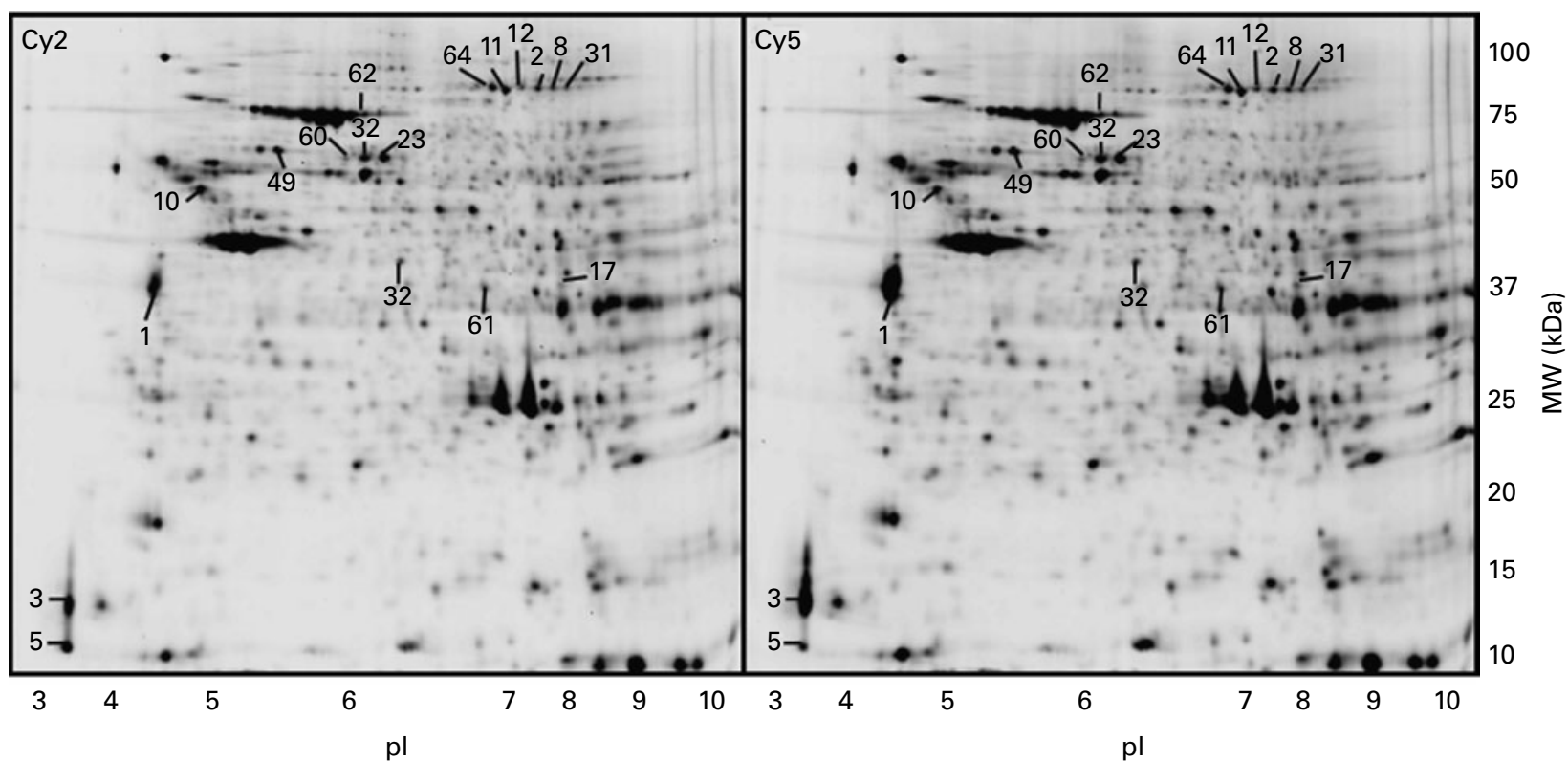

(b)

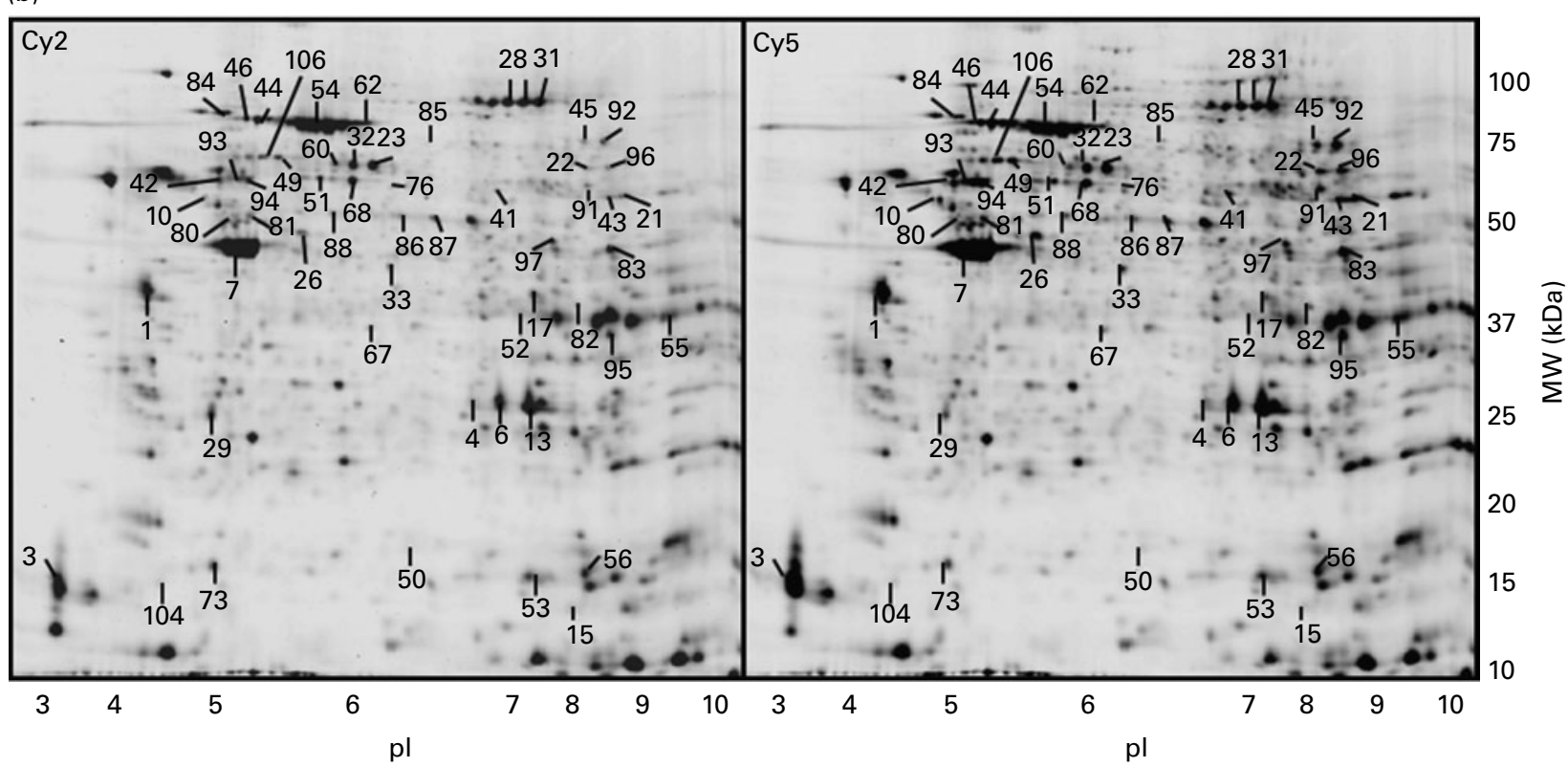

Fig. 2. Gel images showing the differentially expressed spots, control diet $v$. kiwifruit extract-supplemented diet. (a) C57BL/6J, (b) $1 / 10^{-1-}$. Spot identities are listed in Table 5. MW, molecular weight; $\mathrm{pl}, \mathrm{pH}$ of the protein's isoelectric point.

vaccination in otherwise healthy mice, including increased antigen-specific T-cell activation and Ig production ${ }^{(13,14)}$. A pilot human study investigating the effects of an aqueous extract of gold kiwifruit on ex vivo blood samples has found that incubation of blood cells with the KFE enhanced T-cell activation, phagocytosis, oxidative burst and natural killer cell activity $^{(57)}$. Together, these results suggest that the aqueous green KFE may influence immune signalling within the normal colonic tissue; however, the details of this effect are unclear.

Proteasome activity appears to be increased in the colon samples from $1 l 10^{-/-}$mice fed the aqueous green KFE when compared with those fed the control diet, with significantly enriched gene sets and increased protein abundances associated with this pathway. The abundances of two molecular chaperone proteins (heat shock protein 1 and heat shock protein 8) were also reduced in these colon samples, suggesting an overall reduction in cellular stress within these tissues. This would be expected to decrease proteasome activity, rather than molecular weight, the increase identified here. However, these proteins are also involved in TLR signalling ${ }^{(58)}$ and may, instead, have been down-regulated in response to the reduced immune signalling also identified within these colon samples. 


\section{Ethyl acetate gold kiwifruit extract}

In contrast to the anti-inflammatory effects proposed for both aqueous KFE, the transcriptomic results for $1 l 10^{-/-}$mice fed the ethyl acetate gold KFE suggest a pro-inflammatory effect within the colon. The expression levels of genes involved in inflammation (Reg3b and S100a8) or tissue destruction (Mmp10 and Mmp13) are increased within colon samples from $I l 1 O^{-/-}$mice fed the ethyl acetate gold KFE-supplemented diet compared with the control diet (Fig. 1). Gene sets associated with inflammation, cytokine signalling and eicosanoid synthesis are also up-regulated within these mice (Table 4). These outcomes contradict the result of our previous in vitro study where TLR-activated signalling was almost completely inhibited in primary macrophages derived from both $\mathrm{C} 57 \mathrm{BL} / 6 \mathrm{~J}$ and $1110^{-/-}$mice after the gold ethyl acetate KFE treatment ${ }^{(19)}$. However, there were no changes to colon HIS, or protein abundances related to the inflammatory process in these colon samples, indicating that increases in pro-inflammatory immune signalling in $\mathrm{Il1O}^{-/-}$mice in response to ethyl acetate gold KFE supplementation do not result in increased colitis.

A range of signalling pathway gene sets were significantly enriched after the intervention with this KFE, including inflammatory signalling pathways, G-protein-coupled and G-protein-coupled receptor signalling, MAPK and lipid kinase signalling. This is supported by a significant increase in the expression of the key regulatory MAPK protein, p38delta (Mapk13), in $I l 10^{-1-}$ mice fed the ethyl acetate gold KFE when measured by qRT-PCR. These secondary signalling pathways are involved in the development of chronic inflammation within the $1110^{-/-}$mouse colon ${ }^{(59)}$ and may not be involved in the innate immune activation measured by our previous in vitro study. The regulation of these pathways may allow the ethyl acetate gold KFE to increase pro-inflammatory signalling in the colon regardless of their effect on TLR activity. Gene sets related to amino acid, carbohydrate and lipid metabolism show decreased expression levels, indicating lower overall metabolic capacity, potentially because of a reduction in growth factor activity caused by the suppression of these signalling pathways. These findings suggest that ethyl acetate gold KFE may influence the growth factor and inflammatory signalling pathways within the colon.

\section{Ethyl acetate green kiwifruit extract}

The colonic gene expression profiles of both $\mathrm{C} 57 \mathrm{BL} / 6 \mathrm{~J}$ and $\mathrm{Il}_{10} \mathrm{O}^{-1-}$ mice fed diets supplemented with the ethyl acetate green KFE showed reduced expression across sets of genes related to immune function and inflammation compared with expression in colon samples collected from mice fed a KFE-free control diet. The ten gene sets identified in the transcriptomic results for $\mathrm{C} 57 \mathrm{BL} / 6 \mathrm{~J}$ mice are related to aspects of the adaptive immune response, including antigen presentation, IL12 signalling and T-cell activation. However, only two gene sets, each associated with cytokine signalling, were identified as down-regulated in the colons of $1110^{-1-}$ mice. It appears that, while the ethyl acetate green KFE retains some immune-modulating effect within the inflamed $1 l 10^{-1-}$ colon, the putative interaction with the adaptive immune response is lost within this model. This may be due to the type of inflammation that develops. For example, a decrease in IL-12 signalling as seen in the C57BL/6J colon samples may lead to reduced Th1 activation. However, it has been demonstrated that IL-23, but not IL-12, is important for the development of colitis within the $1110^{-/-}$colon $^{(23)}$. Therefore, while a reduction in IL-12 signalling within the $1110^{-1-}$ colon may reduce general pro-inflammatory cytokine signalling (as reported here), it may not be enough to reduce Th17 cell activation within the $1110^{-/-}$colon.

\section{Conclusions}

While dietary intervention with KFE does not reduce colitis in $\mathrm{IllO}^{-/-}$mice, this intervention appears to subtly influence pathways within colonic tissue. In particular, the aqueous gold and green KFE and the ethyl acetate green KFE appear to decrease T-cell-driven adaptive immune signalling within C57BL/6J, but not $1110^{-1-}$, mouse colon samples. These outcomes are in contrast to a previous study where these KFE significantly reduced inflammatory signalling by primary cells isolated from the same $\mathrm{C} 57 \mathrm{BL} / 6 \mathrm{~J}$ and $1110^{-/-}$mouse models ${ }^{(19)}$. This discrepancy highlights the importance of investigating food components identified by cell-based screening assays with appropriate animal models and human clinical studies, as a food that looks promising in vitro may not be effective in vivo. The $1 l 10^{-/-}$mouse studies reported here indicate that clinical studies of KFE in IBD would be inappropriate given our current understanding of their molecular mechanism. However, the changes to adaptive immune signalling, molecular chaperone expression and the overall metabolic effects of KFE identified in the transcriptomic and proteomic data, coupled with in vivo kiwifruit studies conducted by other groups, suggest that KFE may have beneficial activity within the adaptive immune system. This activity may lie in improving the response to vaccination or disease, but does not lie in reducing the inflammatory processes present in the mode of colitis described here. Importantly, two recent human intervention studies have investigated the effects of aqueous gold KFE on the adaptive-immune response ${ }^{(57)}$.

\section{Acknowledgements}

The authors wish to thank Ric Broadhurst, Nerissa Boyes, Kim Oden and Anna Russ for their assistance with the animal experiments, Chrissie Butts for assistance with diet formulation, John Koolaard for the covariate analysis of weight gain, Kate Broadley for training in mRNA extraction and microarray analysis, Matt Punter for training in qRT-PCR, Diane Barraclough for training in two-dimensional electrophoresis and Helge Dzierzon for microarray submission to the Gene Expression Omnibus. Nutrigenomics New Zealand is a collaboration between Plant and Food Research Limited, AgResearch Limited and the University of Auckland, and is 
funded by the New Zealand Foundation for Research, Science and Technology under contract C06X0702.Shelley Edmunds' PhD fellowship was funded by Nutrigenomics New Zealand. All authors have no conflicts of interest. The authors' contributions were as follows: S. J. E. designed, planned and executed all experiments, conducted GSEA and proteomics analysis, and had primary responsibility for the final manuscript. N. C. R., D. R. L. and W. A. L. designed all experiments and provided significant contributions to manuscript writing. M. D. designed the microarray experiments and conducted the microarray quality analysis, LIMMA and other microarray analysis. J. M. C. performed the MS identification of protein spots. S. Z. performed the histology analysis. M. P. G. B. provided training and experimental design for the mouse experiments. Z. P. performed the statistical analysis of all animal experimental data. All authors read and approved the final manuscript.

\section{References}

1. Podolsky DK (2002) Inflammatory bowel disease. New Engl J Med 247, 417-429.

2. Ballegaard M, Bjergstrøm A, Brøndum S, et al. (1997) Selfreported food intolerance in chronic inflammatory bowel cisease. Scand J Gastroenterol 32, 569-571.

3. Ferguson LR, Shelling AN, Browning BL, et al. (2007) Genes, diet and inflammatory bowel disease. Mutat Res 622, 70-83.

4. Ferguson AR \& Ferguson LR (2002) Are kiwifruit really good for you? Acta Hortic 610, 131-138.

5. Nishiyama I (2007) Fruits of the Actinidia genus. In Advances in Food and Nutrition Research, pp. 293-324 [LT Steve, editor]. Waltham, MA: Academic Press.

6. Dawes HM \& Keene JB (1999) Phenolic composition of kiwifruit juice. J Agric Food Chem 47, 2398-2403.

7. Fiorentino A, D'Abrosca B, Pacifico S, et al. (2009) Identification and assessment of antioxidant capacity of phytochemicals from kiwi fruits. J Agric Food Chem 57, 4148-4155.

8. McGhie TK \& Ainge GD (2002) Color in fruit of the genus Actinidia: carotenoid and chlorophyll compositions. J Agric Food Chem 50, 117-121.

9. Motohashi N, Shirataki Y, Kawase M, et al. (2002) Cancer prevention and therapy with kiwifruit in Chinese folklore medicine: a study of kiwifruit extracts. J Ethnopharmacol 81, 357-364.

10. Rush E, Ferguson LR, Cumin M, et al. (2006) Kiwifruit consumption reduces DNA fragility: a randomized controlled pilot study in volunteers. Nutr Res 26, 197-201.

11. Collins AR, Harrington V, Drew J, et al. (2003) Nutritional modulation of DNA repair in a human intervention study. Carcinogenesis 24, 511-515.

12. Iwasawa $H$, Morita $E$, Ueda $H$, et al. (2010) Influence of kiwi fruit on immunity and its anti-oxidant effects in mice. Food Sci Tech Res 16, 135-142.

13. Shu Q, Mendis De Silva U, Chen S, et al. (2008) Kiwifruit extract enhances markers of innate and acquired immunity in a murine model. Food Agric Immunol 19, 149-161.

14. Hunter DC, Denis M, Parlane NA, et al. (2008) Feeding ZESPRI(TM) GOLD Kiwifruit puree to mice enhances serum immunoglobulins specific for ovalbumin and stimulates ovalbumin-specific mesenteric lymph node cell proliferation in response to orally administered ovalbumin. Nutr Res 28, 251-257.
15. Rush EC, Patel M, Plank LD, et al. (2002) Kiwifruit promotes laxation in the elderly. Asia Pac J Clin Nutr 11, 164-168.

16. Chan AOO, Leung G, Tong T, et al. (2007) Increasing dietary fiber intake in terms of kiwifruit improves constipation in Chinese patients. World J Gastroenterol 13, 4771-4775.

17. Murakami A, Ishida H, Kobo K, et al. (2005) Suppressive effects of Okinawan food items on free radical generation from stimulated leukocytes and identification of some active constituents: implications for the prevention of inflammation-associated carcinogenesis. Asian Pac J Cancer P 6 , 437-448.

18. Philpott M, Mackay L, Ferguson LR, et al. (2007) Cell culture models in developing nutrigenomics foods for inflammatory bowel disease. Mutat Res 622, 94-102.

19. Edmunds SJ, Roy NC, Love DR, et al. (2011) Kiwifruit extracts inhibit cytokine production by lipopolysaccharide-activated macrophages, and intestinal epithelial cells isolated from IL10 gene deficient mice. Cell Immunol 270, 70-79.

20. Kuhn R, Lohler J, Rennick D, et al. (1993) Interleukin-10deficient mice develop chronic enterocolitis. Cell $\mathbf{7 5}$, 263-274.

21. Sellon RK, Tonkonogy S, Schultz M, et al. (1998) Resident enteric bacteria are necessary for development of spontaneous colitis and immune system activation in interleukin-10-deficient mice. Infect Immun $\mathrm{J}$ Clin Invest $\mathbf{6 6}$, 5224-5231.

22. Kamada N, Hisamatsu T, Okamoto S, et al. (2008) Unique $\mathrm{CD} 14+$ intestinal macrophages contribute to the pathogenesis of Crohn disease via IL-23/IFN- $\gamma$ axis. J Clin Invest 118, 2269-2280

23. Yen D, Cheung J, Scheerens H, et al. (2006) IL-23 is essential for $\mathrm{T}$ cell-mediated colitis and promotes inflammation via IL-17 and IL-6. J Clin Invest 116, 1310-1316.

24. Montufar-Solis D, Schaefer J, Hicks MJ, et al. (2007) Massive but selective cytokine dysregulation in the colon of IL-10 ${ }^{-/-}$ mice revealed by multiplex analysis. Int Immunol $\mathbf{2 0}$, 141-154.

25. Roy N, Barnett M, Knoch B, et al. (2007) Nutrigenomics applied to an animal model of inflammatory bowel diseases: transcriptomic analysis of the effects of eicosapentaenoic acid- and arachidonic acid-enriched diets. Mutat Res $\mathbf{6 2 2}$, 103-116.

26. Barnett M, McNabb W, Cookson A, et al. (2010) Changes in colon gene expression associated with increased colon inflammation in interleukin-10 gene-deficient mice inoculated with Enterococcus species. BMC Immunol 11, 39.

27. Knoch B, Barnett M, Cooney J, et al. (2010) Molecular characterization of the onset and progression of colitis in inoculated interleukin-10 gene-deficient mice: a role for PPAR $\alpha . P P A R$ Res 2010 (article ID 621069); http://www. hindawi.com/journals/ppar/2010/621069/.

28. Cario E \& Podolsky DK (2000) Differential alteration in intestinal epithelial cell expression of toll-like receptor 3 (TLR3) and TLR4 in inflammatory bowel disease. Infect Immun 68, 7010-7017.

29. Anderson R, Roy N, Barnett M, et al. (2008) Developing smart foods using models of intestinal health. Food Sci Technol Bull 5, 27-38.

30. Reeves PG (1989) AIN-76 diet: should we change the formulation? J Nutr 119, 1081-1082.

31. Reeves PG (1997) Components of the AIN-93 diets as improvements in the AIN-76A diet. J Nutr 127, 838S-841S.

32. Gill H, Shu Q, Lin H, et al. (2001) Protection against translocating Salmonella typhimurium infection in mice by feeding the immuno-enhancing probiotic Lactobacillus rhamnosus strain HN001. Med Microbiol Immunol 190, 97-104. 
33. Park EI, Paisley EA, Mangian HJ, et al. (1997) Lipid level and type alter stearoyl CoA desaturase mRNA abundance differently in mice with distinct susceptibilities to diet-influenced diseases. J Nutr 127, 566-573.

34. Kennedy RJ, Hoper M, Deodhar K, et al. (2000) Interleukin 10-deficient colitis: new similarities to human inflammatory bowel disease. Br J Surg 87, 1346-1351.

35. Gentleman R, Carey V, Bates D, et al. (2004) Bioconductor: open software development for computational biology and bioinformatics. Genome Biol 5, R80.

36. Smyth GK \& Speed T (2003) Normalization of cDNA microarray data. Methods 31, 265-273.

37. Zahurak M, Parmigiani G, Yu W, et al. (2007) Pre-processing Agilent microarray data. BMC Bioinformatics 8, 142.

38. Smyth G (2005) Limma: linear models for microarray data. In Bioinformatics and Computational Biology Solutions using $R$ and Bioconductor, pp. 397-420 [R Gentleman, $\mathrm{V}$ Carey, S Dudoit, R Irizarry and $\mathrm{W}$ Huber, editors]. New York, NY: Springer.

39. Smyth GK (2004) Linear models and empirical Bayes methods for assessing differential expression in microarray experiments. Stat Appl Genet Mol Biol 3 (article 3); http:// www.bepress.com/sagmb/vol3/iss1/art3/.

40. Subramanian A, Kuehn H, Gould J, et al. (2007) GSEA-P: a desktop application for gene set enrichment analysis. Bioinformatics 23, 3251-3253.

41. Subramanian A, Tamayo P, Mootha VK, et al. (2005) Gene set enrichment analysis: a knowledge-based approach for interpreting genome-wide expression profiles. Proc Natl Acad Sci US A 102, 15545-15550.

42. Mootha VK, Lindgren CM, Eriksson K-F, et al. (2003) PGC$1 \alpha$-responsive genes involved in oxidative phosphorylation are coordinately downregulated in human diabetes. Nat Genet 34, 267-273.

43. Vandesompele J, De Preter K, Pattyn F, et al. (2002) Accurate normalization of real-time quantitative RT-PCR data by geometric averaging of multiple internal control genes. Genome Biol 3, research0034-research0034.0011.

44. Knoch B, Barnett M, Zhu S, et al. (2009) Genome-wide analysis of dietary eicosapentaenoic acid- and oleic acid-induced modulation of colon inflammation in interleukin-10 genedeficient mice. J Nutrigenet Nutrigenomics 2, 9-28.

45. Rozen S \& Skaletsky H (1999) Primer3 on the WwW for general users and for biologist programmers. In Bioinformatics Methods and Protocols, pp. 365-386 [S Misener and SA Krawetz, editors]. New York: Humana Press.
46. Pattyn F, Robbrecht P, De Paepe A, et al. (2006) RTPrimerDB: the real-time PCR primer and probe database, major update 2006. Nucleic Acids Res 34, D684-D688.

47. Bradford MM (1976) A rapid and sensitive method for the quantitation of microgram quantities of protein utilizing the principle of protein-dye binding. Anal Biochem $\mathbf{7 2}$, $248-254$

48. Barraclough D, Obenland D, Laing W, et al. (2004) A general method for two-dimensional protein electrophoresis of fruit samples. Postharvest Biol Technol 32, 175-181.

49. Scalbert A \& Williamson G (2000) Dietary intake and bioavailability of polyphenols. J Nutr 130, 2073S-22085.

50. Halliwell B, Rafter J \& Jenner A (2005) Health promotion by flavonoids, tocopherols, tocotrienols, and other phenols: direct or indirect effects? Antioxidant or not? Am J Clin Nutr 81, 268S-2276.

51. Lin H-M, Edmunds SJ, Zhu S, et al. (2011) Metabolomic analysis reveals differences in urinary excretion of kiwifruit-derived metabolites in a mouse model of inflammatory bowel disease. Mol Nutr Food Res (In the Press).

52. Nam D \& Kim S-Y (2008) Gene-set approach for expression pattern analysis. Brief Bioinform 9, 189-197.

53. Shkoda A, Ruiz PA, Daniel H, et al. (2007) Interleukin-10 blocked endoplasmic reticulum stress in intestinal epithelial cells: impact on chronic inflammation. Gastroenterology $\mathbf{1 3 2}$, 190-207.

54. Schröder M \& Kaufman RJ (2005) ER stress and the unfolded protein response. Mutat Res 569, 29-63.

55. Goldberg AL (2003) Protein degradation and protection against misfolded or damaged proteins. Nature $\mathbf{4 2 6}$ 895-899.

56. Feldman DE \& Frydman J (2000) Protein folding in vivo: the importance of molecular chaperones. Curr Opin Struct Biol 10, 26-33.

57. Skinner MA, Loh JMS, Hunter DC, et al. (2011) Gold kiwifruit (Actinidia chinensis 'Hort16A') for immune support. Proc Nutr Soc 70, 276-280.

58. Beg AA (2002) Endogenous ligands of Toll-like receptors: implications for regulating inflammatory and immune responses. Trends Immunol 23, 509-512.

59. Kontoyiannis D, Kotlyarov A, Carballo E, et al. (2001) Interleukin-10 targets p38 MAPK to modulate ARE-dependent TNF mRNA translation and limit intestinal pathology. EMBO J 20, 3760-3770. 